\title{
On the martingale property in stochastic volatility models based on time-homogeneous diffusions*
}

\author{
Carole Bernard ${ }^{\dagger}$ Zhenyu Cui ${ }^{\ddagger}$ and Don McLeish ${ }^{\S}$
}

July 10, 2014

\begin{abstract}
Lions and Musiela (2007) give sufficient conditions to verify when a stochastic exponential of a continuous local martingale is a martingale or a uniformly integrable martingale. Blei and Engelbert (2009) and Mijatović and Urusov (2012c) give necessary and sufficient conditions in the case of perfect correlation $(\rho=1)$. For financial applications, such as checking the martingale property of the stock price process in correlated stochastic volatility models, we extend their work to the arbitrary correlation case $(-1 \leqslant \rho \leqslant 1)$. We give a complete classification of the convergence properties of both perpetual and capped integral functionals of time-homogeneous diffusions and generalize results in Mijatović and Urusov (2012b) (2012c) with direct proofs avoiding the use of separating times (concept introduced by Cherny and Urusov (2004) and extensively used in the proofs of Mijatović and Urusov (2012c)).
\end{abstract}

JEL Classification C02, C63, G12, G13

Keywords: Martingale property, Local martingale, Stochastic volatility, Engelbert-Schmidt zero-one law

${ }^{*}$ C. Bernard acknowledges support from the Natural Sciences and Engineering Research Council of Canada. Z. Cui acknowledges support from the German Academic Exchange Service(DAAD) scholarship at Summer Academy 2012 on "Advanced Stochastic Methods to Model Risk" at Ulm University, Germany, where the paper was presented. D.L. McLeish acknowledges support from the Natural Sciences and Engineering Research Council of Canada. The authors are grateful to an anonymous referee and the associate editor for their careful reading and very helpful suggestions, which improved the paper. The authors thank Antoine Jacquier and seminar participants Christian Benes, Peter Carr, Travis Fisher, Olympia Hadjiliadis, Adam Kolkiewicz, Elena Kosygina, Jay Rosen, David Saunders, Mikhail Urusov, and Jiming Yu for helpful discussions. The usual disclaimer applies.

${ }^{\dagger} \mathrm{C}$. Bernard is with the department of Statistics and Actuarial Science at the University of Waterloo, Email c3bernar@uwaterloo.ca.

${ }^{\ddagger}$ Corresponding author. Z. Cui is with the department of Mathematics at the Brooklyn College of the City University of New York, Email zhenyucui@brooklyn.cuny.edu.

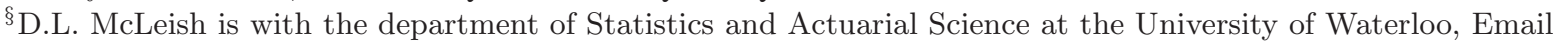
dlmcleis@uwaterloo.ca. 


\section{Introduction}

There are several recent papers proposing sufficient conditions (Lions and Musiela (2007)) or necessary and sufficient conditions (Blei and Engelbert (2009), Delbaen and Shirakawa (2002), Mijatović and Urusov (2012c), Mijatović, Novak and Urusov (2012)) to verify when the stochastic exponential of a continuous local martingale is a true martingale or a uniformly integrable(UI) martingale. A relevant application in finance is to check if the discounted stock price is a true martingale in a general stochastic volatility model with arbitrary correlation.

This problem has been extensively studied and dates back from Girsanov (1960) who poses the problem of deciding whether a stochastic exponential is a true martingale or not. Gikhman and Skorohod (1972), Liptser and Shiryaev (1972), Novikov (1972) and Kazamaki (1977) provide sufficient conditions for the martingale property of a stochastic exponential. Novikov's criterion is easy to apply in practical situations, but it may not always be verified in models in mathematical finance. In the setting of Brownian motions, refer to Kramkov and Shiryaev (1998), Cherny and Shiryaev (2001) and Ruf (2013b) for improvements of the criteria of Novikov (1972) and Kazamaki (1977). For affine processes, similar questions are considered by Kallsen and Shiryaev (2002), Kallsen and Muhle-Karbe (2010), and Mayerhofer, Muhle-Karbe, and Smirnov (2011). Kotani (2006) and Hulley and Platen (2011) obtain necessary and sufficient conditions for a onedimensional regular strong Markov continuous local martingale to be a true martingale. In the strand of stochastic exponentials based on time-homogeneous diffusions, Engelbert and Schmidt (1984) provide analytic conditions for the martingale property, and Stummer (1993) gives further analytic conditions when the diffusion coefficient is the identity. Delbaen and Shirakawa (2002) first provide deterministic criteria to check if a stochastic exponential is a true martingale under a slightly restrictive assumption requiring certain functions to be locally bounded on $(0, \infty)$. Mijatović and Urusov (2012c) removed the restriction of locally boundedness and extend their results utilizing a new tool called separating times introduced in Cherny and Urusov (2004). In the context of stochastic volatility models, Sin (1998), Andersen and Piterbarg (2007), and Lions and Musiela (2007) provide easily verifiable sufficient conditions. Blanchet and Ruf (2012) describe a method to decide on the martingale property of a non-negative local martingale based on weak convergence arguments. Through the study of the classical solutions to the valuation partial differential equation associated with the stochastic volatility model, Bayraktar, Kardaras and Xing (2012) establish a necessary and sufficient condition when the asset price is a martingale. In the context of stochastic differential equations(SDE), Doss and Lenglart (1978) provide a detailed study of their asymptotics and other properties. Ruf (2013a) studies the martingale property of a non-negative local martingale that is given as a nonanticipative functional of a solution to a SDE. A recent paper by Karatzas and Ruf (2013) provides the precise relationship between explosions of one-dimensional stochastic differential equations and the martingale properties of related

stochastic exponentials. For an overview of stochastic exponentials and the related problem of martingale properties, refer to Rheinländer (2010) and the references therein.

This paper makes two contributions to the current literature. First, we provide a complete classification of the convergence or divergence properties of perpetual and capped integral functionals of time-homogeneous diffusions based on the local integrability of certain deterministic test functions. Theorem 3.1 provides similar necessary and sufficient conditions weaker than those in Salminen and Yor (2006), Khoshnevisan, Salminen, and Yor (2006). Mijatović and Urusov (2012a) provide a similar result. Theorem 3.1 permits two absorbing boundaries, while Engelbert and Tittel (2002) assume that there is exactly one absorbing boundary. Theorem 3.2 concerns the 
capped integral functional and, to the best of authors' knowledge, is new. We also extend some results in Mijatović and Urusov (2012b, 2012c) from the case $\rho=1$ to the case $-1 \leqslant \rho \leqslant 1$ (see Proposition 4.1 and Proposition 4.2). Our proofs do not require the concept of separating times introduced by Cherny and Urusov (2004). As examples, we give necessary and sufficient conditions for the (uniformly integrable) martingale property of the stock price in popular stochastic volatility models (Hull-White (1987), (stopped) Heston (1993), Schöbel and Zhu (1999), and 3/2 models).

Section 2 uses the probabilistic setting and technical tools of Ruf (2013b) and Carr, Fisher and Ruf (2014). Section 3 provides a complete classification of the convergence or divergence properties of perpetual and capped integral functionals of time-homogeneous diffusions. The main result of the paper is given in Section 4: we generalize some results in Mijatović and Urusov (2012b, 2012c) to the arbitrary correlation case with new direct proofs. Section 5 studies in detail the martingale properties in four popular stochastic volatility models. Section 6 concludes.

\section{Necessary and sufficient conditions for the martingale property}

\subsection{Probabilistic setup}

Throughout the paper, we fix a time horizon $T \in(0, \infty]$. As in Carr, Fisher and Ruf (2014), we define a stochastic basis by $\left(\Omega, \mathcal{F}_{T},\left\{\mathcal{F}_{t}\right\}_{t \in[0, T]}, P\right)$ with a right-continuous filtration $\left\{\mathcal{F}_{t}\right\}_{t \in[0, T]}$. This basis is assumed rich enough to support the processes described below and satisfies the regularity conditions outlined in Appendix A. For any stopping time $\tau$, we define $\mathcal{F}_{\tau}:=\{A \in$ $\mathcal{F}_{T} \mid A \cap\{\tau \leqslant t\} \in \mathcal{F}_{t}$ for all $\left.t \in[0, T]\right\}$ and $\mathcal{F}_{\tau-}:=\sigma\left(\left\{A \cap\{\tau>t\} \in \mathcal{F}_{T} \mid A \in \mathcal{F}_{t}\right.\right.$ for some $t \in$ $\left.\left.[0, T] \cup \mathcal{F}_{0}\right\}\right)$. In general, non-negative random variables are permitted to take values in the set $[0, \infty]$ and stopping times $\tau$ are permitted to take values in the set $[0, \infty] \cup \mathcal{T}$ for some transfinite time $\mathcal{T}>T$ as in Appendix A of Carr, Fisher and Ruf (2014). In special cases, we will restrict the range.

For an $\mathcal{F}_{t}$-adapted Brownian motion process $W_{t}$, assume that $Y$ satisfies the SDE

$$
d Y_{t}=\mu\left(Y_{t}\right) d t+\sigma\left(Y_{t}\right) d W_{t}, \quad Y_{0}=x_{0},
$$

where $\mu, \sigma: J \rightarrow \mathbb{R}$ are Borel functions, $x_{0} \in J$, and that $\mu, \sigma$ satisfy the Engelbert-Schmidt condition

$$
\forall x \in J, \quad \sigma(x) \neq 0, \quad \text { and } \quad \frac{1}{\sigma^{2}(\cdot)}, \quad \frac{\mu(\cdot)}{\sigma^{2}(\cdot)} \in L_{l o c}^{1}(J) .
$$

Here $L_{l o c}^{1}(J)$ denotes the class of locally integrable functions, i.e. the functions $J \rightarrow \mathbb{R}$ that are integrable on compact subsets of the state space, $J=(\ell, r),-\infty \leqslant \ell<r \leqslant \infty$, of the process $Y=\left(Y_{t}\right)_{t \in[0, T]}$. We set $\bar{J}=[\ell, r]$.

The Engelbert-Schmidt condition (2) guarantees that the SDE (1) has a unique in law weak solution that possibly exits its state space $J$ (see Theorem 5.15, page 341, Karatzas and Shreve (1991)). Denote the possible exit time ${ }^{1}$ of $Y$ from its state space by $\zeta$, i.e. $\zeta=\inf \left\{u>0, Y_{u} \notin J\right\}$, $P$-a.s. which means that on $\{\zeta=\infty\}$ the trajectories of $Y$ do not exit $J, P$-a.s., and on $\{\zeta<\infty\}$, $\lim _{t \rightarrow \zeta} Y_{t}=r$ or $\lim _{t \rightarrow \zeta} Y_{t}=\ell, P$-a.s.. Observe that $Y$ is defined such that it stays at its exit

\footnotetext{
${ }^{1}$ Refer to Karatzas and Ruf (2013) for a detailed study of the distribution of this exit time in a one-dimensional time-homogeneous diffusion setting.
} 
point, which means that $\ell$ and $r$ are absorbing boundaries. The following terminology will be used: "Y may exit the state space $J$ at $r$ " means $P\left(\zeta<\infty, \lim _{t \rightarrow \zeta} Y_{t}=r\right)>0$.

Then we introduce a standard Brownian motion $W^{(2)}$ independent of $(Y, W)$. Let $Z=$ $\left(Z_{t}\right)_{t \in[0, T]}$ denote the (discounted) stock price with $Z_{0}=1$, and define

$$
Z_{t}=\exp \left\{\rho \int_{0}^{t \wedge \zeta} b\left(Y_{u}\right) d W_{u}+\sqrt{1-\rho^{2}} \int_{0}^{t \wedge \zeta} b\left(Y_{u}\right) d W_{u}^{(2)}-\frac{1}{2} \int_{0}^{t \wedge \zeta} b^{2}\left(Y_{u}\right) d u\right\}, \quad t \in[0, \infty),
$$

where $b: J \rightarrow \mathbb{R}$ is a Borel function, and the constant correlation satisfies $-1 \leqslant \rho \leqslant 1$.

Denote $W^{(1)}=\rho W .+\sqrt{1-\rho^{2}} W^{(2)}$, we have

$$
Z_{t}=\exp \left\{\int_{0}^{t \wedge \zeta} b\left(Y_{u}\right) d W_{u}^{(1)}-\frac{1}{2} \int_{0}^{t \wedge \zeta} b^{2}\left(Y_{u}\right) d u\right\}, \quad t \in[0, \infty)
$$

and it is easy to verify that $Z$ and $Y$ satisfy the following system of SDEs

$$
\begin{aligned}
& d Z_{t}=Z_{t} b\left(Y_{t}\right) d W_{t}^{(1)}, \quad Z_{0}=1, \\
& d Y_{t}=\mu\left(Y_{t}\right) d t+\sigma\left(Y_{t}\right) d W_{t}, \quad Y_{0}=x_{0} .
\end{aligned}
$$

The Borel sigma algebra $\mathcal{B}(\mathbb{R})$ in $\mathbb{R}$ is the smallest $\sigma$-algebra that contains the open intervals of $\mathbb{R}$. In what follows, $\lambda(\cdot)$ denotes the Lebesgue measure on $\mathcal{B}(\mathbb{R})$. We require that ${ }^{2} \lambda(x \in(\ell, r)$ : $\left.b^{2}(x)>0\right)>0$, and assume the following local integrability condition

$$
\forall x \in J, \quad \sigma(x) \neq 0, \quad \text { and } \quad \frac{b^{2}(\cdot)}{\sigma^{2}(\cdot)} \in L_{l o c}^{1}(J)
$$

Remark 2.1. In the literature (e.g. Andersen and Piterbarg (2007)), there is a more general class of stochastic volatility models where the (discounted) stock price has a non-linear diffusion coefficient in $Z$. For example, a general model is as follows

$$
\begin{aligned}
& d Z_{t}=Z_{t}^{\alpha} b\left(Y_{t}\right) \mathbb{1}_{t \in[0, \zeta)} d W_{t}^{(1)}, \quad Z_{0}=1, \\
& d Y_{t}=\mu\left(Y_{t}\right) \mathbb{1}_{t \in[0, \zeta)} d t+\sigma\left(Y_{t}\right) \mathbb{1}_{t \in[0, \zeta)} d W_{t}, \quad Y_{0}=x_{0},
\end{aligned}
$$

where $W_{t}^{(1)}$ and $W_{t}$ are standard $\mathcal{F}_{t}$-Brownian motions, with $\mathbb{E}\left[d W_{t}^{(1)} d W_{t}\right]=\rho d t . \rho$ is the constant correlation coefficient and $-1 \leqslant \rho \leqslant 1$. Here $1 \leqslant \alpha \leqslant 2$. The difficulty of dealing with this model lies mainly in obtaining an explicit representation of $Z$ in terms of functionals of only $Y$. Thus, in this paper, we only focus on model (5).

Lemma 2.1. (Mijatović and Urusov (2012c)). Assume conditions (2) and (6), and $0<t<\infty$. Then

$$
\int_{0}^{t} b^{2}\left(Y_{u}\right) d u<\infty \text { P-a.s. on }\{t<\zeta\}
$$

\footnotetext{
${ }^{2}$ Note that this is the same condition as in Mijatović and Urusov (2012b, 2012c), and Cherny and Urusov (2006).
} 
Fix an arbitrary constant $c \in J$ and introduce the scale function $s(\cdot)$ of the SDE (1) under $P$

$$
s(x):=\int_{c}^{x} \exp \left\{-\int_{c}^{y} \frac{2 \mu}{\sigma^{2}}(u) d u\right\} d y, \quad x \in \bar{J} .
$$

The following result and its proof can be found in Cherny and Urusov (2006), here translated into our notation.

Lemma 2.2. (Lemma 5.7, page 149 of Cherny and Urusov (2006)). Assume conditions (2) and (6) for the $S D E(1)$, and $s(\ell)=-\infty, s(r)=\infty$. Then $\int_{0}^{\infty} b^{2}\left(Y_{u}\right) d u=\infty, P$-a.s.

\subsection{Properties of non-negative continuous local martingales}

In this section, we fix a time horizon $T \in(0, \infty]$, and work under the canonical probability space $\left(\Omega, \mathcal{F}_{T},\left(\mathcal{F}_{t}\right)_{t \in[0, T]}, P\right)$. This space must be rich enough to support processes with distributions described below, and the filtration $\left(\mathcal{F}_{t}\right)_{t \in[0, T]}$ must satisfy additional conditions outlined in Appendix A. We begin by applying some results from Ruf (2013b) and Carr, Fisher and Ruf (2014) concerning non-negative continuous local martingales to time-homogeneous diffusions as in (4). Ruf (2013b) does not specify the form of the continuous local martingale $\left(L_{t}\right)_{t \in[0, T)}$, which is, in our setting

$$
L_{t}=\int_{0}^{t \wedge \zeta} b\left(Y_{u}\right) d W_{u}^{(1)}
$$

To cast the setting of Ruf (2013b) into the current notation, the process in (4) under $P$ can be rewritten as $Z_{t}=\mathcal{E}\left(L_{t}\right)=\exp \left(L_{t}-\langle L\rangle_{t} / 2\right)$ where $L_{t}$ in (8) is a continuous local martingale under $P$.

Lemma 2.3. (Lemma 1, Ruf (2013b)) Assume conditions (2) and (6) for the SDE (1). Under $P$, consider the continuous local martingale $\left(L_{t}\right)_{t \in[0, T]}$ given in (8), and its quadratic variation $\langle L\rangle_{t}=\int_{0}^{t \wedge \zeta} b^{2}\left(Y_{u}\right) d u$. For a predictable positive stopping time $0<\tau \leqslant \infty$, define $Z_{t}=\mathcal{E}\left(L_{t}\right), t \in$ $[0, \tau)$. Then the random variable $Z_{\tau}:=\lim _{t \uparrow \tau} Z_{t}$ exists, is non-negative and satisfies

$$
\left\{\int_{0}^{\tau \wedge \zeta} b^{2}\left(Y_{u}\right) d u<\infty\right\}=\left\{Z_{\tau}>0\right\}, \quad P-a . s .
$$

As an application of Lemma 2.3, we have the following result.

Corollary 2.1. Assume $e^{3}$ conditions (2) and (6) for the SDE (1). Under P, with the process $Z$ defined in (4), for $t \in[0, T]$

$$
\left\{Z_{t}=0\right\}=\left\{\zeta \leqslant t, \int_{0}^{\zeta} b^{2}\left(Y_{u}\right) d u=\infty\right\}, \quad P \text {-a.s. }
$$

Proof. From Lemma 2.3,

$$
\left\{Z_{t}=0\right\}=\left\{\int_{0}^{t \wedge \zeta} b^{2}\left(Y_{u}\right) d u=\infty\right\}, \quad P \text {-a.s. }
$$

\footnotetext{
${ }^{3}$ This is stated without proof after equation (7) on page 4, Mijatović and Urusov (2012c), and after equation (2.4) on page 228, Mijatović and Urusov (2012b). Here we provide a proof.
} 
From Lemma 2.1, $P\left(\int_{0}^{t \wedge \zeta} b^{2}\left(Y_{u}\right) d u<\infty\right)=P\left(\int_{0}^{t} b^{2}\left(Y_{u}\right) d u<\infty\right)=1$ on the set $\{t<\zeta, t \in[0, T]\}$. Therefore

$$
\left\{Z_{t}=0\right\}=\left\{\zeta \leqslant t, \int_{0}^{t \wedge \zeta} b^{2}\left(Y_{u}\right) d u=\infty\right\}, \quad P \text {-a.s. }
$$

In the following, for notation convenience, denote $T_{\infty}:=R$ and $T_{0}:=S$ as the first hitting times to $\infty$ and 0 respectively by $Z$, where $R$ and $S$ are defined in Section 2.1. Both may take values in $[0, \infty] \cup \mathcal{T}$. The next result is Theorem 2.1 of Carr, Fisher and Ruf (2014) and given in our notation.

Proposition 2.1. (Theorem 2.1, of Carr, Fisher and Ruf (2014)). Consider the canonical probability space $\left(\Omega, \mathcal{F}_{T},\left(\mathcal{F}_{t}\right)_{t \in[0, T]}, P\right)$, with the process $Z$ defined in (4) (so that $Z_{0}=1$ ) and assume conditions $(2)$ and $(6)$. Then there exists a unique probability measure $\widetilde{P}$ on $\left(\Omega, \mathcal{F}_{T_{\infty}-}\right)$ such that, for any stopping time $0<\nu<\infty$,

$$
\widetilde{P}\left(A \cap\left\{T_{\infty}>\nu \wedge T\right\}\right)=\mathbb{E}^{P}\left[\mathbb{1}_{A} Z_{\nu \wedge T}\right]
$$

for all $A \in \mathcal{F}_{\nu \wedge T}$.

(2) for all non-negative $\mathcal{F}_{\nu \wedge T}$-measurable random variables $U$ taking values in $[0, \infty]$,

$$
\mathbb{E}^{\widetilde{P}}\left[U \mathbb{1}_{\left\{T_{\infty}>\nu \wedge T\right\}}\right]=\mathbb{E}^{P}\left[U Z_{\nu \wedge T} \mathbb{1}_{\left\{T_{0}>\nu \wedge T\right\}}\right]
$$

and, with $\widetilde{Z}_{t}=\frac{1}{Z_{t}} \mathbb{1}_{\left\{T_{\infty}>t\right\}^{5}}$,

$$
\mathbb{E}^{P}\left[U \mathbb{1}_{\left\{T_{0}>\nu \wedge T\right\}}\right]=\mathbb{E}^{\widetilde{P}}\left[U \widetilde{Z}_{\nu \wedge T}\right]
$$

(3) $Z$ is a uniformly integrable $P$ martingale on $[0, T]$ if and only if

$$
\widetilde{P}\left(T_{\infty}>T\right)=1 \text {. }
$$

Notice that from (9), for any stopping time $\nu<T, \widetilde{P}\left(Z_{\nu}=0\right)=0$ so that the measure $\widetilde{P}$ assigns zero mass to paths $Z_{t}$ that hit 0 . The condition (12) is equivalent to

$$
\widetilde{P}\left(\sup _{t \in(0, T]} Z_{t}<\infty\right)=\widetilde{P}\left(\inf _{t \in(0, T]} \widetilde{Z}_{t}>0\right)=1 \text { or } \widetilde{P}\left(\sup _{t \in[0, T]} Z_{t}=\infty\right)=0
$$

Proposition 2.2. (1) Under $P$, for $t \in\left[0, T_{0}\right)$, define the continuous $P$-local martingale $L_{t}$ as in (8). Then under $\widetilde{P}$, for $t \in\left[0, T_{\infty}\right), \widetilde{L}_{t}:=L_{t}-\langle L\rangle_{t}=\int_{0}^{t \wedge \zeta} b\left(Y_{u}\right) d W_{u}^{(1)}-\int_{0}^{t \wedge \zeta} b^{2}\left(Y_{u}\right) d u$ is a continuous $\widetilde{P}$-local martingale.

(2) Under $\widetilde{P}$, for $t \in\left[0, T_{\infty}\right)$

$$
\widetilde{Z}_{t}=\mathcal{E}\left(-\widetilde{L}_{t}\right)=\exp \left\{-\int_{0}^{t \wedge \zeta} b\left(Y_{u}\right) d W_{u}^{(1)}+\frac{1}{2} \int_{0}^{t \wedge \zeta} b^{2}\left(Y_{u}\right) d u\right\} .
$$

\footnotetext{
${ }^{4}$ Theorem 2.1, page 6 of Carr, Fisher and Ruf (2014) is a general result for non-negative local martingales. See also Ruf (2013b) for a similar result for continuous non-negative local martingales.

${ }^{5}$ By definition this is 0 whenever $t \geqslant T_{\infty}$ even if $Z_{t}=0$.
} 
Proof. For statement (1), we need to show that $\widetilde{L}_{t}=\int_{0}^{t \wedge \zeta} b\left(Y_{u}\right) d W_{u}^{(1)}-\int_{0}^{t \wedge \zeta} b^{2}\left(Y_{u}\right) d u$ is a $\widetilde{P}_{-}$ local-martingale on $\left[0, T_{\infty}\right)$. Recall $R_{n}$ is the first hitting time of $Z_{t}$ to the level $n$, and put $\tau_{n}=R_{n} \wedge n$ for all $n \in \mathbb{N}$. We will show that $\widetilde{L}_{t \wedge \tau_{n}}=\int_{0}^{t \wedge \zeta \wedge \tau_{n}} b\left(Y_{u}\right) d W_{u}^{(1)}-\int_{0}^{t \wedge \zeta \wedge \tau_{n}} b^{2}\left(Y_{u}\right) d u$ is a $\widetilde{P}$-local martingale. This follows from the Girsanov theorem (Ch.VIII, Theorem 1.4 in Revuz and Yor (1999)), the facts that $\widetilde{P}<<P$ on $\mathcal{F}_{\tau_{n}}$ and $\widetilde{P}\left(\lim _{n \rightarrow \infty} \tau_{n}=T_{\infty}\right)=1$.

For statement (2), under $\widetilde{P}$, for $t<T_{\infty}$

$$
\begin{aligned}
\widetilde{Z}_{t} & =\exp \left\{-\int_{0}^{t \wedge \zeta} b\left(Y_{u}\right) d W_{u}^{(1)}+\frac{1}{2} \int_{0}^{t \wedge \zeta} b^{2}\left(Y_{u}\right) d u\right\} \\
& =\exp \left\{-\int_{0}^{t \wedge \zeta} b\left(Y_{u}\right) d W_{u}^{(1)}+\int_{0}^{t \wedge \zeta} b^{2}\left(Y_{u}\right) d u-\frac{1}{2} \int_{0}^{t \wedge \zeta} b^{2}\left(Y_{u}\right) d u\right\} \\
& =\mathcal{E}\left(-\widetilde{L}_{t}^{*}\right) .
\end{aligned}
$$

Now we seek to determine the SDE satisfied by $Y$ under $\widetilde{P}$.

Proposition 2.3. Assume conditions (2) and (6) for the SDE (1). Under $\widetilde{P}$, for $-1 \leqslant \rho \leqslant 1$, the diffusion $Y$ satisfies the following SDE up to $\zeta$

$$
d Y_{t}=\left(\mu\left(Y_{t}\right)+\rho b\left(Y_{t}\right) \sigma\left(Y_{t}\right)\right) \mathbb{1}_{t \in[0, \zeta)} d t+\sigma\left(Y_{t}\right) \mathbb{1}_{t \in[0, \zeta)} d \widetilde{W}_{t}, \quad Y_{0}=x_{0} .
$$

Proof. Consider the system of SDEs in (5), from the Cholesky decomposition, $d W_{t}^{(1)}=\rho d W_{t}+$ $\sqrt{1-\rho^{2}} d W_{t}^{(2)}$, where $W$ and $W^{(2)}$ are standard independent Brownian motions under $P$. Define for $t \in[0, T]$

$$
\widetilde{W}_{t}:= \begin{cases}W_{t}-\rho \int_{0}^{t} b\left(Y_{u}\right) d u, & \text { if } t<\zeta, \\ W_{\zeta}-\rho \int_{0}^{\zeta} b\left(Y_{u}\right) d u+\widetilde{\beta}_{t-\zeta}, & \text { if } t \geqslant \zeta,\end{cases}
$$

where $\widetilde{\beta}$ is a standard $\widetilde{P}$-Brownian motion independent of $W$ with $\widetilde{\beta}_{0}=0$.

Define $\xi_{n}=\zeta \wedge \tau_{n}$, where $\tau_{n}=R_{n} \wedge n$ and consider the process $\widetilde{W}$ up to $\xi_{n}$. Since $\mathcal{F}_{\xi_{n}} \subset \mathcal{F}_{\tau_{n}}$, it follows from Proposition 2.1 that $\widetilde{P}$ restricted to $\mathcal{F}_{\xi_{n}}$ is absolutely continuous with respect to $P$ restricted to $\mathcal{F}_{\xi_{n}}$ for $n \in \mathbb{N}$. Then from Girsanov Theorem (Ch.VIII, Theorem 1.12, page 331 of Revuz and Yor (1999))

$$
\begin{aligned}
\widetilde{W}_{t} & :=W_{t}-\left\langle W_{t}, \int_{0}^{t} b\left(Y_{u}\right) d W_{u}^{(1)}\right\rangle \\
& =W_{t}-\left\langle W_{t}, \rho \int_{0}^{t} b\left(Y_{u}\right) d W_{u}\right\rangle-\left\langle W_{t}, \sqrt{1-\rho^{2}} \int_{0}^{t} b\left(Y_{u}\right) d W_{u}^{(2)}\right\rangle \\
& =W_{t}-\rho \int_{0}^{t} b\left(Y_{u}\right) d u,
\end{aligned}
$$

is a $\widetilde{P}$-Brownian motion for $t \in\left[0, \xi_{n}\right)$ and $n \in \mathbb{N}$. It is easy to see from the construction (14), the finite dimensional distributions of $\widetilde{W}$ are those of a Brownian motion under $\widetilde{P}$ on $\left[0, \xi_{n}\right)$. Thus $Y$ is governed by the following SDE under $\widetilde{P}$ for $t \in\left[0, \xi_{n}\right)$

$$
\begin{aligned}
d Y_{t} & =\mu\left(Y_{t}\right) d t+\sigma\left(Y_{t}\right)\left(d \widetilde{W}_{t}+\rho b\left(Y_{t}\right) d t\right) \\
& =\left(\mu\left(Y_{t}\right)+\rho b\left(Y_{t}\right) \sigma\left(Y_{t}\right)\right) d t+\sigma\left(Y_{t}\right) d \widetilde{W}_{t}, \quad Y_{0}=x_{0} .
\end{aligned}
$$


The result will follow from the following lemma which shows that $\xi_{n}=\zeta \wedge R_{n} \wedge n \rightarrow \zeta \wedge T_{\infty}=\zeta$, $P$-a.s.

Lemma 2.4. Assume conditions (2) and (6), then $\zeta \leqslant T_{0} \wedge T_{\infty}, P$-a.s. and $\widetilde{P}$-a.s.

Proof. We prove by contradiction that $P\left(T_{0} \wedge T_{\infty}<\zeta\right)=0$. Suppose that $T_{\infty}<\zeta$ with positive probability so that for some $t, P\left(T_{\infty}<t<\zeta\right)>0$. Since $T_{\infty}<t, P\left(Z_{t}=\infty\right)>0$. By Lemma 2.1 ,

$$
P\left(\int_{0}^{t} b^{2}\left(Y_{u}\right) d u<\infty, Z_{t}=\infty\right)>0
$$

Note that $Z_{t}=\exp \left(L_{t}-\frac{1}{2}\langle L\rangle_{t}\right)=\infty$ if and only if $L_{t}=\infty$. By the Dambis-Dubins-Schwartz theorem (Ch.V, Theorem 1.6, Revuz and Yor (1999)), for some Brownian motion $B$ on an extended probability space, we can write $L_{t}-\frac{1}{2}\langle L\rangle_{t}=\langle L\rangle_{t}\left(\frac{B\langle L\rangle_{t}}{\langle L\rangle_{t}}-\frac{1}{2}\right)$ and from the continuity of the Brownian motion, $P\left(\langle L\rangle_{t}<\infty, L_{t}-\frac{1}{2}\langle L\rangle_{t}=\infty\right)=P\left(\langle L\rangle_{t}<\infty, B_{\langle L\rangle_{t}}=\infty\right)=0$ so that

$$
P\left(\int_{0}^{t} b^{2}\left(Y_{u}\right) d u<\infty, Z_{t}=\infty\right)=P\left(\langle L\rangle_{t}<\infty, L_{t}-\frac{1}{2}\langle L\rangle_{t}=\infty\right)=0
$$

contradicting (16). Similarly suppose that, for some $t, P\left(T_{0}<t<\zeta\right)>0$. Then $P\left(Z_{t}=0\right)>0$ and since $t<\zeta$, from Lemma 2.1,

$$
P\left(\int_{0}^{t} b^{2}\left(Y_{u}\right) d u<\infty, Z_{t}=0\right)>0
$$

contradicting Lemma 2.3. We have thus shown that $P\left(T_{\infty}<\zeta\right)=P\left(T_{0}<\zeta\right)=0$. To demonstrate a similar statement under the probability measure $\widetilde{P}$, note that $\widetilde{P}$ is a probability measure on $\left(\Omega, \mathcal{F}_{R-}\right)$ such that, for a stopping time $R_{n}$,

$$
\widetilde{P}\left(\zeta>T_{0} \wedge R_{n}\right)=\widetilde{P}\left(\left\{\zeta>T_{0} \wedge R_{n}\right\} \cap\left\{T_{\infty}>R_{n}\right\}\right)=\mathbb{E}^{P}\left[\mathbb{1}_{\left\{\zeta>T_{0} \wedge R_{n}\right\}} Z_{R_{n}}\right]=0
$$

since $\left\{\zeta>T_{0} \wedge R_{n}\right\}$ is a $\mathcal{F}_{R_{n}}$ measurable event. The last equality holds since $P\left(\zeta>T_{0} \wedge R_{n}\right)=0$. Then by monotone convergence

$$
\widetilde{P}\left(\zeta>T_{0} \wedge T_{\infty}\right)=\lim _{n \rightarrow \infty} \widetilde{P}\left(\zeta>T_{0} \wedge R_{n}\right)=0
$$

In view of Lemma 2.4 and the definition of $Z_{t}$ in (4), there are only three possibilities almost surely under the measures $P$ and $\widetilde{P}$ :

$$
\zeta=T_{0}<T_{\infty}=\mathcal{T} \quad \text { or } \quad \zeta=T_{\infty}<T_{0}=\mathcal{T} \quad \text { or } \quad \zeta<T_{0}=T_{\infty}=\mathcal{T}
$$

In order to verify $\mathbb{E}^{P}\left[Z_{T}\right]=1$ for $T \in[0, \infty]$, the equivalent condition in Proposition 2.1, (3) can be transformed into a condition related to integral functionals of $Y$ under $\widetilde{P}$ as shown in the following proposition.

Proposition 2.4. Assume ${ }^{6}$ conditions (2) and (6), and $T \in[0, \infty]$. Then $Z_{t}$ is a (uniformly integrable) P-martingale for $t \in[0, T]$, i.e. $\mathbb{E}^{P}\left[Z_{T}\right]=1$, if and only if $\widetilde{P}\left(\int_{0}^{T \wedge \zeta} b^{2}\left(Y_{u}\right) d u<\infty\right)=$ 1.

\footnotetext{
${ }^{6}$ A similar result for the general setting of multi-dimensional diffusions appears in Theorem 1 of Ruf (2013a).
} 
Proof. By Proposition 2.1 (3), we have a uniformly integrable martingale satisfying $\mathbb{E}^{P}\left[Z_{T}\right]=1$ if and only if

$$
\widetilde{P}\left(T_{\infty}>T\right)=\widetilde{P}\left(0<\inf _{t \in[0, T]} \widetilde{Z}_{t}\right)=1
$$

But by Proposition $2.2(2)$, under the measure $\widetilde{P}$

$$
\widetilde{Z}_{t}=\mathcal{E}\left(-\widetilde{L}_{t}\right)=\exp \left\{-\int_{0}^{t \wedge \zeta} b\left(Y_{u}\right) d W_{u}^{(1)}+\frac{1}{2} \int_{0}^{t \wedge \zeta} b^{2}\left(Y_{u}\right) d u\right\}
$$

is a continuous local martingale and for a stopping time $\tau=T \wedge T_{\infty} \wedge \zeta=T \wedge \zeta$, by Lemma 2.3, $\left\{\widetilde{Z}_{\tau}>0\right\}=\left\{\int_{0}^{\tau \wedge \zeta} b^{2}\left(Y_{u}\right) d u<\infty\right\}$. The result follows.

Remark 2.2. Since $\widetilde{P}\left(\int_{0}^{T \wedge \zeta} b^{2}\left(Y_{u}\right) d u<\infty\right)=\lim _{q \rightarrow 0} E^{\widetilde{P}}\left[e^{-q \int_{0}^{T \wedge \zeta} b^{2}\left(Y_{u}\right) d u}\right]$ is the right limit of the Laplace transform $\mathcal{L}(q)$ of $\int_{0}^{T \wedge \zeta} b^{2}\left(Y_{u}\right) d u$ at 0 under the measure $\widetilde{P}$ (and defining $\mathcal{L}(0)=1$ ), we have the alternative formulation of Proposition 2.4 that $Z_{t}$ is a (uniformly integrable) $P$ martingale on $[0, T]$, i.e. $\mathbb{E}^{P}\left[Z_{T}\right]=1$, if and only if $\mathcal{L}(q)$ is right continuous at 0 .

\section{Classification of convergence properties of integral functionals of time-homogeneous diffusions}

The Engelbert-Schmidt zero-one law was initially proved in the Brownian motion case (see Engelbert and Schmidt (1981) or Proposition 3.6.27, page 216 of Karatzas and Shreve (1991)). Engelbert and Tittel (2002) obtain a generalized Engelbert-Schmidt type zero-one law for the integral functional $\int_{0}^{t} f\left(X_{s}\right) d s$, where $f$ is a non-negative Borel function and $X$ is a strong Markov continuous local martingale. In an expository paper, Mijatović and Urusov (2012a) consider the case of a one-dimensional time-homogeneous diffusion and the zero-one law is given in their Theorem 2.11. They provide two proofs that circumvent the use of Jeulin's lemma ${ }^{7}$. Through stochastic time-change, Cui (2014) proposes a new proof under a slightly stronger assumption.

Recall the scale function $s(\cdot)$ defined in $(7)$, and introduce the following test functions for $x \in \bar{J}$, with a constant $c \in J$.

$$
\begin{aligned}
v(x) & :=\int_{c}^{x}(s(x)-s(y)) \frac{2}{s^{\prime}(y) \sigma^{2}(y)} d y, \\
v_{b}(x) & :=\int_{c}^{x}(s(x)-s(y)) \frac{2 b^{2}(y)}{s^{\prime}(y) \sigma^{2}(y)} d y .
\end{aligned}
$$

Note that if $s(\infty)=\infty$, then $v(\infty)=\infty$ and $v_{b}(\infty)=\infty$ by the definition in (17). Define $\widetilde{s}(\cdot)$, $\widetilde{v}(\cdot)$ and $\widetilde{v}_{b}(\cdot)$ similarly based on the $\operatorname{SDE}(13)$ under $\widetilde{P}$. Throughout this section, we assume that $\lambda\left(x \in(\ell, r): b^{2}(x)>0\right)>0$, which is assumed in Mijatović and Urusov (2012a).

We have the following Engelbert-Schmidt type zero-one law for the SDE (1) under $P$, which is Theorem 2.11 of Mijatović and Urusov (2012a) with $f(\cdot)=b^{2}(\cdot)$ using our notation.

Proposition 3.1. (Engelbert-Schmidt type zero-one law for a time-homogeneous diffusion, Theorem 2.11 of Mijatović and Urusov (2012a))

\footnotetext{
${ }^{7}$ The first proof is based on William's theorem (Ch.VII, Corollary 4.6, page 317, Revuz and Yor (1999)). The second proof is based on the first Ray-Knight theorem (Ch.XI, Theorem 2.2, page 455, Revuz and Yor (1999)).
} 
Assume conditions (2), (6) and $s(r)<\infty$.

(i) If $v_{b}(r)<\infty$, then $\int_{0}^{\zeta} b^{2}\left(Y_{u}\right) d u<\infty, P$-a.s. on $\left\{\lim _{t \rightarrow \zeta} Y_{t}=r\right\}$.

(ii)If $v_{b}(r)=\infty$, then $\int_{0}^{\zeta} b^{2}\left(Y_{u}\right) d u=\infty, P$-a.s. on $\left\{\lim _{t \rightarrow \zeta} Y_{t}=r\right\}$.

Analogous results on the set $\left\{\lim _{t \rightarrow \zeta} Y_{t}=\ell\right\}$ can be similarly stated. Clearly the above proposition has a counterpart for the SDE (13) under $\widetilde{P}$ for the end points $r$ and $\ell$.

The following result is Proposition 5.5.22 on page 345 of Karatzas and Shreve (1991) using our notation. It classifies possible exit behaviors of the process $Y$ at the boundaries of its state space $J$ under $P$.

Proposition 3.2. (Proposition 5.5.22, Karatzas and Shreve (1991)) Assume condition (2). Let $Y$ be a weak solution of (1) in $J$ under $P$, with nonrandom initial condition $Y_{0}=x_{0} \in J$. Distinguish four cases:

(a) If $s(\ell)=-\infty$ and $s(r)=\infty, P(\zeta=\infty)=P\left(\sup _{0 \leqslant t<\infty} Y_{t}=r\right)=P\left(\inf _{0 \leqslant t<\infty} Y_{t}=\ell\right)=1$.

(b) If $s(\ell)>-\infty$ and $s(r)=\infty, P\left(\lim _{t \rightarrow \zeta} Y_{t}=\ell\right)=P\left(\sup _{0 \leqslant t<\zeta} Y_{t}<r\right)=1$.

(c) If $s(\ell)=-\infty$ and $s(r)<\infty, P\left(\lim _{t \rightarrow \zeta} Y_{t}=r\right)=P\left(\inf _{0 \leqslant t<\zeta} Y_{t}>\ell\right)=1$.

(d) If $s(\ell)>-\infty$ and $s(r)<\infty, P\left(\lim _{t \rightarrow \zeta} Y_{t}=\ell\right)=1-P\left(\lim _{t \rightarrow \zeta} Y_{t}=r\right)=\frac{s(r)-s\left(x_{0}\right)}{s(r)-s(\ell)}$. Note that $0<\frac{s(r)-s\left(x_{0}\right)}{s(r)-s(\ell)}<1$.

Analogous results also hold for the $\operatorname{SDE}(13)$ under $\widetilde{P}$.

Remark 3.1. In the conditions $(b),(c)$ and $(d)$ above, we make no claim concerning the finiteness of $\zeta$. See Remark 5.5.23 on page 345 of Karatzas and Shreve (1991). Note that conditions (b) and (c) are consequences of the expression in condition $(d)$ by letting either $s(r)=\infty$ or $s(\ell)=-\infty$.

Similar to the statements in Proposition 3.2, for the study of the convergence or divergence properties of integral functionals of time-homogeneous diffusions, we distinguish the following four exhaustive and disjoint cases under $P$ :

- Case (1): $s(\ell)=-\infty, s(r)=\infty$.

- Case (2): $s(\ell)=-\infty, s(r)<\infty$.

- Case (3): $s(\ell)>-\infty, s(r)=\infty$.

- Case (4): $s(\ell)>-\infty, s(r)<\infty$.

Further divide each case above into the following subcases based on the finiteness of $v_{b}(r)$ and $v_{b}(\ell)$ as defined in $(17)$ :

Case (2)(i): $s(\ell)=-\infty, s(r)<\infty, v_{b}(r)=\infty$.

Case (2)(ii): $s(\ell)=-\infty, s(r)<\infty, v_{b}(r)<\infty$.

Case (3)(i): $s(\ell)>-\infty, s(r)=\infty, v_{b}(\ell)=\infty$.

Case (3)(ii): $s(\ell)>-\infty, s(r)=\infty, v_{b}(\ell)<\infty$.

Case (4)(i): $s(\ell)>-\infty, s(r)<\infty, v_{b}(r)=\infty, v_{b}(\ell)=\infty$.

Case (4)(ii): $s(\ell)>-\infty, s(r)<\infty, v_{b}(r)<\infty, v_{b}(\ell)=\infty$.

Case (4)(iii): $s(\ell)>-\infty, s(r)<\infty, v_{b}(r)=\infty, v_{b}(\ell)<\infty$.

Case (4)(iv): $s(\ell)>-\infty, s(r)<\infty, v_{b}(r)<\infty, v_{b}(\ell)<\infty$.

Define

$$
\varphi_{t}:=\int_{0}^{t} b^{2}\left(Y_{u}\right) d u
$$

for $t \in[0, \zeta]$. Recall that $b^{2}(\cdot)$ is a non-negative Borel function, thus $\varphi_{t}$ is a non-decreasing function for $t \in[0, \zeta]$. Because $\varphi_{t}$ is an integral, it is continuous for $t \in[0, \zeta)$, and is left continuous at $t=\zeta$. We now apply the Engelbert-Schmidt type zero-one law under $P$ as in Proposition 3.1 to 
determine whether $P\left(\varphi_{\zeta}<\infty\right)=1$ or $P\left(\varphi_{\zeta}=\infty\right)=1$ in each of the cases above. We first prove two lemmas.

Lemma 3.1. Assume ${ }^{8}$ conditions (2) and (6), then " $v_{b}(\ell)=\infty$ and $v_{b}(r)=\infty$ " are necessary and sufficient for $P\left(\varphi_{\zeta}=\infty\right)=1$.

Proof. For the sufficiency, assume $v_{b}(r)=\infty$ and $v_{b}(\ell)=\infty$ and consider the following four distinct cases:

- Case (1): $s(\ell)=-\infty, s(r)=\infty$. From Proposition $3.2($ a), we have $P(\zeta=\infty)=1$. This, combined with Lemma 2.2 implies $P\left(\varphi_{\zeta}=\infty\right)=1$.

- Case (2): $s(\ell)=-\infty, s(r)<\infty$. From Proposition $3.2(\mathrm{c}), P\left(\lim _{t \rightarrow \zeta} Y_{t}=r\right)=1$. Since $v_{b}(r)=\infty$, then from Proposition $3.1 P\left(\varphi_{\zeta}=\infty\right)=P\left(\varphi_{\zeta}=\infty, \lim _{t \rightarrow \zeta} Y_{t}=r\right)$ and from Proposition 3.2, $P\left(\varphi_{\zeta}=\infty, \lim _{t \rightarrow \zeta} Y_{t}=r\right)=P\left(\lim _{t \rightarrow \zeta} Y_{t}=r\right)=1$.

- Case (3): $s(\ell)>-\infty, s(r)=\infty$. The proof is similar to Case (2) above by switching the roles of $\ell$ and $r$, and applying Proposition 3.2 (b) and Proposition 3.1.

- Case (4): $s(\ell)>-\infty, s(r)<\infty$. From Proposition $3.2(\mathrm{~d}), 0<p=P\left(\lim _{t \rightarrow \zeta} Y_{t}=r\right)<1$. Since $v_{b}(r)=\infty$ and $v_{b}(\ell)=\infty$, from Proposition 3.1

$$
\begin{aligned}
P\left(\varphi_{\zeta}\right. & =\infty)=P\left(\varphi_{\zeta}=\infty, \lim _{t \rightarrow \zeta} Y_{t}=r\right)+P\left(\varphi_{\zeta}=\infty, \lim _{t \rightarrow \zeta} Y_{t}=\ell\right) \\
& =P\left(\lim _{t \rightarrow \zeta} Y_{t}=r\right)+P\left(\lim _{t \rightarrow \zeta} Y_{t}=\ell\right)=1 .
\end{aligned}
$$

For the necessity, we only need to prove the contrapositive statement: "If at least one of $v_{b}(\ell)$ or $v_{b}(r)$ is finite, then $P\left(\varphi_{\zeta}=\infty\right)<1$." Note that case (a) of Proposition 3.2 is ruled out here so that we are assured that $P\left(\lim _{t \rightarrow \zeta} Y_{t}=r\right)+P\left(\lim _{t \rightarrow \zeta} Y_{t}=\ell\right)=1$. Without loss of generality, assume that $v_{b}(\ell)<\infty$, because the case $v_{b}(r)<\infty$ can be similarly proved. Then

$$
\begin{aligned}
P\left(\varphi_{\zeta}\right. & =\infty)=P\left(\varphi_{\zeta}=\infty, \lim _{t \rightarrow \zeta} Y_{t}=\ell\right)+P\left(\varphi_{\zeta}=\infty, \lim _{t \rightarrow \zeta} Y_{t}=r\right) \\
& =P\left(\varphi_{\zeta}=\infty, \lim _{t \rightarrow \zeta} Y_{t}=r\right) \\
& \leqslant P\left(\lim _{t \rightarrow \zeta} Y_{t}=r\right)
\end{aligned}
$$

where the second line follows since from Proposition 3.1, $P\left(\varphi_{\zeta}=\infty, \lim _{t \rightarrow \zeta} Y_{t}=\ell\right)=0$. There are now two possibilities for $s(r)$. If $s(r)=\infty$, since $s(\ell)>-\infty$, we have $P\left(\lim _{t \rightarrow \zeta} Y_{t}=r\right)=0$ from Proposition $3.2(\mathrm{~b})$. Alternatively, if $s(r)<\infty$, since also $s(\ell)>-\infty$ we have from Proposition $3.2(\mathrm{~d}), 0<p=P\left(\lim _{t \rightarrow \zeta} Y_{t}=r\right)<1$. In both cases $P\left(\lim _{t \rightarrow \zeta} Y_{t}=r\right)<1$, thus $P\left(\varphi_{\zeta}=\infty\right)<1$, and the necessity follows.

Lemma 3.2. Assume $e^{9}$ conditions (2) and (6), and $s(\ell)>-\infty, s(r)<\infty$, then " $v_{b}(\ell)<\infty$ and $v_{b}(r)<\infty$ " are necessary and sufficient for $P\left(\varphi_{\zeta}<\infty\right)=1$.

Proof. With $s(\ell)>-\infty$ and $s(r)<\infty$, denote $p=P\left(\lim _{t \rightarrow \zeta} Y_{t}=r\right)=1-P\left(\lim _{t \rightarrow \zeta} Y_{t}=\ell\right)$. From Proposition $3.2(\mathrm{~d}), 0<p<1$.

For the sufficiency, assume that $v_{b}(\ell)<\infty$ and $v_{b}(r)<\infty$ hold. We aim to prove that $P\left(\varphi_{\zeta}<\infty\right)=1$ where $\phi_{\zeta}=\int_{0}^{\zeta} b^{2}\left(Y_{u}\right) d u$ according to its definition (18).

\footnotetext{
${ }^{8}$ Lemma 5.7, page 149 of Cherny and Urusov (2006) is a one-sided version of the current result, namely, "if $s(\ell)=\infty$ and $s(r)=\infty$ (which implies $v_{b}(\ell)=\infty$ and $\left.v_{b}(r)=\infty\right)$, then $P\left(\varphi_{\zeta}=\infty\right)=1$."

${ }^{9}$ Theorem 2.11 on page 61 of Mijatović and Urusov (2012a) give a similar result.
} 
From Proposition 3.1, $P\left(\varphi_{\zeta}<\infty, \lim _{t \rightarrow \zeta} Y_{t}=r\right)=P\left(\lim _{t \rightarrow \zeta} Y_{t}=r\right)$ and $P\left(\varphi_{\zeta}<\infty, \lim _{t \rightarrow \zeta} Y_{t}=\right.$ $\ell)=P\left(\lim _{t \rightarrow \zeta} Y_{t}=\ell\right)$. Then

$$
\begin{aligned}
P\left(\varphi_{\zeta}<\infty\right) & =P\left(\varphi_{\zeta}<\infty, \lim _{t \rightarrow \zeta} Y_{t}=r\right)+P\left(\varphi_{\zeta}<\infty, \lim _{t \rightarrow \zeta} Y_{t}=\ell\right) \\
& =P\left(\lim _{t \rightarrow \zeta} Y_{t}=r\right)+P\left(\lim _{t \rightarrow \zeta} Y_{t}=\ell\right)=1 .
\end{aligned}
$$

For the necessity, we only need to prove the contrapositive argument: "If at least one of $v_{b}(\ell)$ and $v_{b}(r)$ is infinite, then $P\left(\varphi_{\zeta}<\infty\right)<1$." Without loss of generality, assume that $v_{b}(r)=\infty$, because the case $v_{b}(\ell)=\infty$ can be similarly proved. From Proposition 3.1, $P\left(\varphi_{\zeta}<\infty, \lim _{t \rightarrow \zeta} Y_{t}=r\right)=0$, and

$$
\begin{aligned}
P\left(\varphi_{\zeta}<\infty\right) & =P\left(\varphi_{\zeta}<\infty, \lim _{t \rightarrow \zeta} Y_{t}=r\right)+P\left(\varphi_{\zeta}<\infty, \lim _{t \rightarrow \zeta} Y_{t}=\ell\right) \\
& =P\left(\varphi_{\zeta}<\infty, \lim _{t \rightarrow \zeta} Y_{t}=\ell\right) \\
& \leqslant P\left(\lim _{t \rightarrow \zeta} Y_{t}=\ell\right)<1, \quad \text { from Proposition } 3.2 .
\end{aligned}
$$

Thus the necessity follows.

We now give a detailed study of the function $\varphi_{t}, t \in[0, \zeta]$ under $P$ using the Engelbert-Schmidt type zero-one law. Theorem 3.1 completely characterizes the convergence or divergence property of $\varphi_{t}, t \in[0, \zeta]$, and several results from the literature are one-sided versions of it: Theorem 3.1 (i) is Lemma 2.1, which is stated and proved after equation (9) on page 5 of Mijatović and Urusov (2012c). Theorem 2 on page 3 of Khoshnevisan, Salminen, and Yor (2006) provides ${ }^{10}$ the necessary and sufficient conditions for $P\left(\varphi_{\zeta}<\infty\right)=1$, which corresponds to Theorem 3.1 (ii). However, they make use of the stochastic time change and Itō's lemma in their proof, and thus need to assume the twice differentiability of a function $g(\cdot)$ defined in their paper. Our proof is based on Engelbert-Schmidt type zero-one laws of Mijatović and Urusov (2012a), and our weaker assumptions concern the local integrability of certain deterministic functions. Under these assumptions, Mijatović and Urusov (2012a) give a result similar to Theorem 3.1 (ii) (in their Theorem 2.11). In a parallel paper, Engelbert and Tittel (2002) consider a strong Markov continuous local martingale and is broader in scope. As a comparison, their Proposition 3.7 gives necessary and sufficient conditions for the integral functional to be convergent or divergent, but assume in Proposition 3.7 that the process $X$ has exactly one absorbing point whereas in our setting and that of Mijatović and Urusov (2012a), it is assumed that the process $Y$ can be absorbed at either boundary $\ell$ or $r$.

Theorem 3.1. Under conditions (2) and (6), the following properties for $\varphi_{t}, t \in[0, \zeta]$ hold:

(i) $\varphi_{t}<\infty P$-a.s. on $\{0 \leqslant t<\zeta\}$.

(ii) $P\left(\varphi_{\zeta}<\infty\right)=1$ if and only if at least one of the following conditions is satisfied:

(a) $v_{b}(r)<\infty$ and $s(\ell)=-\infty$,

(b) $v_{b}(\ell)<\infty$ and $s(r)=\infty$,

(c) $v_{b}(r)<\infty$ and $v_{b}(\ell)<\infty$.

(iii) $P\left(\varphi_{\zeta}=\infty\right)=1$ if and only if $v_{b}(r)=\infty$ and $v_{b}(\ell)=\infty$.

\footnotetext{
${ }^{10}$ Salminen and Yor (2006) give similar conditions for a Brownian motion with drift, and Khoshnevisan, Salminen, and Yor (2006) extend it to time-homogeneous diffusions.
} 
We summarize the results of Theorem 3.1 in Table 1 hereafter. Note that $P\left(\varphi_{\zeta}<\infty\right)=$ $P\left(Z_{\infty}>0\right)$ always holds by taking $\tau=\infty$ in Lemma 2.3, and the last two columns in Table 1 agree.

Proof. Statement (i) follows from Lemma 2.1. For statement (ii), the detailed proof for each of the cases in Table 1 is as follows:

- In Case (1), $s(\ell)=-\infty$ and $s(r)=\infty$ and so from Lemma 2.2, $P\left(\varphi_{\zeta}=\infty\right)=1$.

- In Case (2), $s(\ell)=-\infty$ and $s(r)<\infty$ and so from Proposition 3.2, $P\left(\lim _{t \rightarrow \zeta} Y_{t}=r\right)=1$. There are two possible subcases. First, in Case (2)(i), $v_{b}(r)<\infty$ and it follows from Lemma 2.1 that $P\left(\varphi_{\zeta}=\infty\right)=1$. In Case (2)(ii), since $v_{b}(r)<\infty$, we have from Lemma 3.1 that $\varphi_{\zeta}<\infty$ a.s. on the set $\left\{\lim _{t \rightarrow \zeta} Y_{t}=r\right\}$. Moreover, from Proposition 3.2, $P\left(\lim _{t \rightarrow \zeta} Y_{t}=r\right)=1$. It follows that $P\left(\varphi_{\zeta}<\infty\right)=1$.

- In Case (3), $s(\ell)>-\infty$ and $s(r)=\infty$ and so from Proposition 3.2, $P\left(\lim _{t \rightarrow \zeta} Y_{t}=\ell\right)=1$. Again there are two possible subcases, but they are the reverse of cases in (2); Case (3)(i) is exactly the reverse of (2)(i) with $\ell$ and $r$ interchanged and similarly, Case (3)(ii) is exactly the reverse of (2)(ii) so the proofs in Case (2) suffice.

- In Case (4): $s(\ell)>-\infty$ and $s(r)<\infty$. Then, from Proposition 3.2, $1>p=P\left(\lim _{t \rightarrow \zeta} Y_{t}=\right.$ $r)=1-P\left(\lim _{t \rightarrow \zeta} Y_{t}=\ell\right)>0$. For individual subcases, in Case 4(i), Lemma 3.1 implies $P\left(\varphi_{\zeta}=\right.$ $\infty)=1$. In Case (4)(ii), Proposition 3.1 implies that $P\left(\varphi_{\zeta}=\infty\right)<1$ so that $P\left(\varphi_{\zeta}<\infty\right)>0$. By Lemma 3.2, we have $P\left(\varphi_{\zeta}<\infty\right)<1$. Case (4) (iii) is exactly the reverse of (4)(ii) with $\ell$ and $r$ interchanged so the proof follows using this substitution. And finally, for Case (4)(iv), $P\left(\varphi_{\zeta}<\infty\right)=1$ follows from Lemma 3.2. Therefore, we have the three distinct behaviors for $P\left(\varphi_{\zeta}<\infty\right)$ as outlined in Table 1 . The necessity follows by examination of Table 1.

\begin{tabular}{|c|c|c|c|c|c|c|c|}
\hline Case & & $s(\ell)$ & $s(r)$ & $v_{b}(\ell)$ & $v_{b}(r)$ & $P\left(\varphi_{\zeta}<\infty\right)$ & $P\left(Z_{\infty}>0\right)$ \\
\hline$(1)$ & & $-\infty$ & $\infty$ & $\infty$ & $\infty$ & 0 & 0 \\
\hline \multirow{2}{*}{$(2)$} & (i) & $-\infty$ & $<\infty$ & $\infty$ & $\infty$ & 0 & 0 \\
\hline & (ii) & $-\infty$ & $<\infty$ & $\infty$ & $<\infty$ & 1 & 1 \\
\hline \multirow{2}{*}{ (3) } & (i) & $>-\infty$ & $\infty$ & $\infty$ & $\infty$ & 0 & 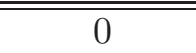 \\
\hline & (ii) & $>-\infty$ & $\infty$ & $<\infty$ & $\infty$ & 1 & 1 \\
\hline \multirow{4}{*}{ (4) } & (i) & $>-\infty$ & $<\infty$ & $\infty$ & $\infty$ & 0 & 0 \\
\hline & (ii) & $>-\infty$ & $<\infty$ & $\infty$ & $<\infty$ & $(0,1)^{*}$ & $(0,1)^{*}$ \\
\hline & (iii) & $>-\infty$ & $<\infty$ & $<\infty$ & $\infty$ & $(0,1)^{*}$ & $(0,1)^{*}$ \\
\hline & (iv) & $>-\infty$ & $<\infty$ & $<\infty$ & $<\infty$ & 1 & 1 \\
\hline
\end{tabular}

Table 1: Table indicating the positivity of the stock price and the finiteness of $\varphi_{\zeta}$. (* indicates that the probability lies in the open interval $(0,1))$.

Similar results as Theorem 3.1 hold under $\widetilde{P}$, and the results are summarized in Table 2 . Note that $\mathbb{E}^{P}\left[Z_{\infty}\right]=\widetilde{P}\left(\varphi_{\zeta}<\infty\right)$ from Proposition 2.4, and the second-to-last and third-to-last columns in Table 2 are equal. 


\begin{tabular}{cc|c|c|c|c|c|c|c}
\multicolumn{2}{l|}{ Case } & $\widetilde{s}(\ell)$ & $\widetilde{s}(r)$ & $\widetilde{v}_{b}(\ell)$ & $\widetilde{v}_{b}(r)$ & $\widetilde{P}\left(\varphi_{\zeta}<\infty\right)$ & $\mathbb{E}^{P}\left(Z_{\infty}\right)$ & UI Mart. \\
\hline \multicolumn{2}{l|}{$(1)$} & $-\infty$ & $\infty$ & $\infty$ & $\infty$ & 0 & $<1$ & No \\
\hline \hline \multirow{2}{*}{$(2)$} & (i) & $-\infty$ & $<\infty$ & $\infty$ & $\infty$ & 0 & $<1$ & No \\
\cline { 2 - 9 } & (ii) & $-\infty$ & $<\infty$ & $\infty$ & $<\infty$ & 1 & 1 & Yes \\
\hline \hline \multirow{2}{*}{$(3)$} & (i) & $>-\infty$ & $\infty$ & $\infty$ & $\infty$ & 0 & $<1$ & No \\
\cline { 2 - 9 } & (ii) & $>-\infty$ & $\infty$ & $<\infty$ & $\infty$ & 1 & 1 & Yes \\
\hline \hline \multirow{5}{*}{$(4)$} & (i) & $>-\infty$ & $<\infty$ & $\infty$ & $\infty$ & 0 & $<1$ & No \\
\cline { 2 - 8 } & (ii) & $>-\infty$ & $<\infty$ & $\infty$ & $<\infty$ & $(0,1)^{*}$ & $<1$ & No \\
\cline { 2 - 8 } & (iii) & $>-\infty$ & $<\infty$ & $<\infty$ & $\infty$ & $(0,1)^{*}$ & $<1$ & No \\
\cline { 2 - 8 } & (iv) & $>-\infty$ & $<\infty$ & $<\infty$ & $<\infty$ & 1 & 1 & Yes \\
\hline
\end{tabular}

Table 2: Table indicating $\mathbb{E}^{P}\left(\mathbf{Z}_{\infty}\right)$ and the uniform integrability of $Z$. (*indicates that the probability lies in the open interval $(0,1))$

The following result provides necessary and sufficient conditions for $P\left(\varphi_{\zeta \wedge T}<\infty\right)=1$, for $T \in(0, \infty)$.

Theorem 3.2. Assume conditions (2) and (6).

$$
P\left(\varphi_{\zeta \wedge T}<\infty\right)=P\left(\int_{0}^{\zeta \wedge T} b^{2}\left(Y_{u}\right) d u<\infty\right)=1
$$

for all $T \in(0, \infty)$ if and only if at least one of the following conditions is satisfied:

(a) $v(\ell)=v(r)=\infty$

(b) $v_{b}(r)<\infty$ and $v(\ell)=\infty$,

(c) $v_{b}(\ell)<\infty$ and $v(r)=\infty$,

(d) $v_{b}(r)<\infty$ and $v_{b}(\ell)<\infty$.

Proof. The conditions state that $\left(\{v(\ell)=\infty\}\right.$ or $\left.\left\{v_{b}(\ell)<\infty\right\}\right)$ and $\left(\{v(r)=\infty\}\right.$ or $\left.\left\{v_{b}(r)<\infty\right\}\right)$. For a given $T<\infty$, define the events $A_{T}=\left\{\varphi_{\zeta \wedge T}<\infty\right\}, A=\left\{\varphi_{\zeta}<\infty\right\}$ and $B=\{\zeta<\infty\}$. Notice that the sets $A_{T} \cap B$ form a decreasing sequence of sets (as $T \rightarrow \infty$ through a countable set) so that $\bigcap_{T}\left(A_{T} \cap B\right)=A \cap B$. Therefore,

$$
P\left(A_{T} \cap B\right) \downarrow P(A \cap B) \text { as } T \rightarrow \infty .
$$

Moreover, from Theorem 3.1 (i), for each $T<\infty$,

$$
P\left(A_{T} \cap \bar{B}\right)=P(\bar{B}) .
$$

We wish to find necessary and sufficient conditions for $P\left(A_{T}\right)=1$ for all $T<\infty$. In view of (19) and (20), this is equivalent to the condition

$$
\begin{aligned}
& P\left(A_{T} \cap B\right)+P(\bar{B})=1 \text { for all } T \text { or } \\
& P(A \cap B)+P(\bar{B})=1 \text { or } P(B \cap \bar{A})=0 .
\end{aligned}
$$

In other words, we seek necessary and sufficient conditions to ensure that

$$
P\left(\zeta<\infty, \varphi_{\zeta}=\infty\right)=0 .
$$


We first show the sufficiency of the above conditions. Condition (a) and Feller's test for explosions implies $P(\zeta<\infty)=0$ and so $(22)$ follows. $P\left(\varphi_{\zeta}=\infty\right)=0$ is implied in the cases 2(ii), 3(ii) or 4(iv) of Table 1. These conditions are special cases of conditions (b), (c) and (d) as indicated in Table 3 below.

\begin{tabular}{c|c|c} 
Case & implies & Cases in Table 1 \\
\hline (b) $v_{b}(r)<\infty$ and $v(\ell)=\infty$ & $s(r)<\infty$ & $2(\mathrm{ii}), 4(\mathrm{ii}), 4(\mathrm{iv})$ \\
\hline (c) $v_{b}(\ell)<\infty$ and $v(r)=\infty$ & $s(\ell)>-\infty$ & $3(\mathrm{ii}), 4(\mathrm{iii}), 4(\mathrm{iv})$ \\
\hline (d) $v_{b}(r)<\infty$ and $v_{b}(\ell)<\infty$ & $s(r)<\infty, s(\ell)>-\infty$ & $4(\mathrm{iv})$ \\
\hline
\end{tabular}

Table 3: Correspondence between conditions (b), (c) and (d) for the sufficiency case and cases in Table 1.

It remains to show (22) in case 4(ii), i.e. $v(\ell)=\infty, s(\ell)>-\infty, v_{b}(r)<\infty, s(r)<\infty$ and in case 4(iii), i.e. $v_{b}(\ell)<\infty, s(\ell)>-\infty, v(r)=\infty, s(r)<\infty$. By interchanging the role of $\ell$ and $r$, it suffices to show the first of these. By Proposition 3.1, $\varphi_{\zeta}<\infty P$-a.s. on the set $\left\{\lim _{t \rightarrow \zeta} Y_{t}=r\right\}$ or

$$
P\left(\varphi_{\zeta}=\infty, \lim _{t \rightarrow \zeta} Y_{t}=r\right)=0 .
$$

From Feller's test of explosions, $v(\ell)<\infty$ if and only if $P\left(\zeta<\infty, \lim _{t \rightarrow \zeta} Y_{t}=\ell\right)>0$, and so in this case $v(\ell)=\infty$ implies

$$
P\left(\zeta<\infty, \lim _{t \rightarrow \zeta} Y_{t}=\ell\right)=0
$$

It follows that

$$
\begin{aligned}
P\left(\zeta<\infty, \varphi_{\zeta}=\infty\right) & =P\left(\zeta<\infty, \varphi_{\zeta}=\infty, \lim _{t \rightarrow \zeta} Y_{t}=\ell\right)+P\left(\zeta<\infty, \varphi_{\zeta}=\infty, \lim _{t \rightarrow \zeta} Y_{t}=r\right) \\
& \leqslant P\left(\zeta<\infty, \lim _{t \rightarrow \zeta} Y_{t}=\ell\right)+P\left(\varphi_{\zeta}=\infty, \lim _{t \rightarrow \zeta} Y_{t}=r\right)=0 .
\end{aligned}
$$

For the necessity, we wish to show the contrapositive: if $\left\{v(\ell)<\infty\right.$ and $\left.v_{b}(\ell)=\infty\right\}$ OR $\left\{v(r)<\infty\right.$ and $\left.v_{b}(r)=\infty\right\}$ (i.e. at least one of the two boundaries, $v$ is finite and $v_{b}$ infinite), then (22) fails, that is

$$
P\left(\zeta<\infty, \varphi_{\zeta}=\infty\right)>0 .
$$

The contrapositive is consistent with Table 1, cases 2(i), 3(i), 4(i), 4(ii), 4(iii) as indicated in Table 4 below.

\begin{tabular}{c|c|c|c} 
Contrapositive Case & implies & & Cases in Table 1 \\
\hline$\{v(\ell)<\infty\}$ and $\left\{v_{b}(\ell)=\infty\right\}$ & $s(\ell)>-\infty$ & Consistent with & $3(\mathrm{i}), 4(\mathrm{i}), 4(\mathrm{ii})$ \\
\hline$\{v(r)<\infty\}$ and $\left\{v_{b}(r)=\infty\right\}$ & $s(r)<\infty$ & Consistent with & $2(\mathrm{i}), 4(\mathrm{i}), 4(\mathrm{iii})$ \\
\hline
\end{tabular}

Table 4: Correspondence between the two conditions from the contrapositive case and cases in Table 1. 
Consider the first row above when $v(\ell)<\infty, v_{b}(\ell)=\infty, s(\ell)>-\infty$. By Feller's test, $v(\ell)<\infty$ implies $P\left(\zeta<\infty, \lim _{t \rightarrow \zeta} Y_{t}=\ell\right)>0$ and by Proposition 3.1, since $v_{b}(\ell)=\infty, \varphi_{\zeta}=\infty P$-a.s. on the set $\left\{\lim _{t \rightarrow \zeta} Y_{t}=\ell\right\}$ and

$$
P\left(\zeta<\infty, \varphi_{\zeta}=\infty\right) \geqslant P\left(\zeta<\infty, \varphi_{\zeta}=\infty, \lim _{t \rightarrow \zeta} Y_{t}=\ell\right)=P\left(\zeta<\infty, \lim _{t \rightarrow \zeta} Y_{t}=\ell\right)>0
$$

The proof in the second case $v(r)<\infty, v_{b}(r)=\infty$ follows once again by interchanging the roles of $\ell$ and $r$.

Similarly statements as Theorem 3.2 hold under $\widetilde{P}$ with SDE (13).

\section{Generalization of some results in Mijatović and Urusov}

In this section, we generalize the main results in Mijatović and Urusov (2012b, 2012c) and provide new unified proofs without the concepts of "separating times". Note that Mijatović and Urusov $(2012 \mathrm{~b}, 2012 \mathrm{c}$ ) work in the $\rho=1$ case, and we generalize it to the arbitrary correlation case.

Consider the stochastic exponential $Z$ defined in (4). The following proposition provides the necessary and sufficient condition for $Z_{T}$ to be a $P$-martingale for all $T \in(0, \infty)$, when $-1 \leqslant \rho \leqslant 1$. Note that Theorem 2.1 in Mijatović and Urusov (2012c) is the case $\rho=1$ of the following proposition.

Proposition 4.1. Assume conditions (2) and (6), then for all $T \in(0, \infty), \mathbb{E}^{P}\left[Z_{T}\right]=1$ if and only if at least one of the conditions (1)-(4) below is satisfied:

(1) $\widetilde{v}(\ell)=\widetilde{v}(r)=\infty$,

(2) $\widetilde{v}_{b}(r)<\infty$ and $\widetilde{v}(\ell)=\infty$,

(3) $\widetilde{v}_{b}(\ell)<\infty$ and $\widetilde{v}(r)=\infty$,

(4) $\widetilde{v}_{b}(r)<\infty$ and $\widetilde{v}_{b}(\ell)<\infty$.

Proof. From Proposition 2.4, for all $T \in(0, \infty), \mathbb{E}^{P}\left[Z_{T}\right]=1$ if and only if $\widetilde{P}\left(\int_{0}^{\zeta \wedge T} b^{2}\left(Y_{u}\right) d u<\right.$ $\infty)=1$. Then the statement follows from Theorem 3.2 applied to $\widetilde{P}$.

We have the following necessary and sufficient condition for $Z$ to be a uniformly integrable $P$-martingale on $[0, \infty]$, when $-1 \leqslant \rho \leqslant 1$. Note that Theorem 2.3 of Mijatović and Urusov (2012c) proves the case $\rho=1$ of the following proposition.

Proposition 4.2. Assume conditions (2) and (6), then $\mathbb{E}^{P}\left[Z_{\infty}\right]=1$ if and only if at least one of the conditions $\left(A^{\prime}\right)-\left(D^{\prime}\right)$ below is satisfied:

$\left(A^{\prime}\right) b=0$ a.e. on $J$ with respect to the Lebesgue measure,

$\left(B^{\prime}\right) \widetilde{v}_{b}(r)<\infty$ and $\widetilde{s}(\ell)=-\infty$,

$\left(C^{\prime}\right) \widetilde{v}_{b}(\ell)<\infty$ and $\widetilde{s}(r)=\infty$

$\left(D^{\prime}\right) \widetilde{v}_{b}(r)<\infty$ and $\widetilde{v}_{b}(\ell)<\infty$.

Proof. From Proposition 2.4, $\mathbb{E}^{P}\left[Z_{\infty}\right]=1$ if and only if $\widetilde{P}\left(\int_{0}^{\zeta} b^{2}\left(Y_{u}\right) d u<\infty\right)=1$. Condition $\left(A^{\prime}\right)$ is a trivial case and it is easy to verify. From Theorem 3.1 applied to $\widetilde{P}$ and the classification in Table $2, \mathbb{E}^{P}\left[Z_{\infty}\right]=1$ if and only if at least one of the conditions $\left(B^{\prime}\right),\left(C^{\prime}\right)$ or $\left(D^{\prime}\right)$ holds.

Here we generalize some results in Mijatović and Urusov (2012b) to the arbitrary correlation case and provide new proofs without the concept of separating times. Precisely, Theorem 2.1 of Mijatović and Urusov (2012b) is the case $\rho=1$ of the following proposition. 
Proposition 4.3. Assume conditions (2) and (6), then for all $T \in(0, \infty), Z_{T}>0$ P-a.s. if and only if at least one of the conditions ${ }^{11}$ (1)-(4) below is satisfied:

(1) $v(\ell)=v(r)=\infty$,

(2) $v_{b}(r)<\infty$ and $v(\ell)=\infty$,

(3) $v_{b}(\ell)<\infty$ and $v(r)=\infty$,

(4) $v_{b}(r)<\infty$ and $v_{b}(\ell)<\infty$.

Proof. From Lemma 2.3, for all $T \in(0, \infty), Z_{T}>0, P$-a.s. if and only if $P\left(\int_{0}^{\zeta \wedge T} b^{2}\left(Y_{u}\right) d u<\infty\right)=$ 1. Then the statement follows from Theorem 3.2.

Note that Theorem 2.3 of Mijatović and Urusov (2012b) proves the case $\rho=1$ of the following proposition.

Proposition 4.4. Let the functions $\mu, \sigma$ and b satisfy conditions (2.1), (2.3) and (2.5) of $M i$ jatović and Urusov (2012b) (equivalently conditions (2) and (6) in this paper), and let $Y$ be a (possibly explosive) solution of the SDE (1) under $P$, with $Z$ defined in (4), then $Z_{\infty}>0, P$-a.s. if and only if at least one of the conditions (I)-(IV) below is satisfied:

(I) $b=0$ a.e. on $J$ with respect to the Lebesgue measure,

(II) $v_{b}(r)<\infty$ and $s(\ell)=-\infty$,

(III) $v_{b}(\ell)<\infty$ and $s(r)=\infty$,

$(I V) v_{b}(r)<\infty$ and $v_{b}(\ell)<\infty$.

Proof. Condition (I) is a trivial case and it is easy to verify. From Lemma 2.3, $Z_{\infty}>0, P$ a.s. if and only if $P\left(\int_{0}^{\zeta} b^{2}\left(Y_{s}\right) d s<\infty\right)=1$. Then the proof follows from Theorem 3.1 and the classification in Table 1.

Note that Theorem 2.5 of Mijatović and Urusov (2012b) is a special case of the following proposition when $\rho=1$.

Proposition 4.5. Let the functions $\mu, \sigma$ and $b$ satisfy conditions (2.1), (2.3) and (2.5) of Mijatović and Urusov (2012b) (equivalently conditions (2) and (6) in this paper), and let $Y$ be a (possibly explosive) solution of the SDE (1) under $P$, with $Z$ defined in (4). Then $Z_{\infty}=0$, P-a.s. if and only if both conditions (i) and (ii) below are satisfied:

(i) $b$ is not identically zero with respect to Lebesgue measure on $(\ell, r)$,

(ii) $v_{b}(\ell)=v_{b}(r)=\infty$.

Proof. Condition (i) is a trivial case and it is easy to verify. From Lemma $2.3, Z_{\infty}=0$, $P$-a.s. if and only if $P\left(\int_{0}^{\zeta} b^{2}\left(Y_{u}\right) d u=\infty\right)=P\left(\varphi_{\zeta}=\infty\right)=1$. From Theorem 3.1 (iii), this is equivalent to checking the condition (ii) here.

\section{Examples of correlated stochastic volatility models}

In this section, we apply the results in Section 4 to the study of martingale properties of (discounted) stock prices ${ }^{12}$ in four popular correlated stochastic volatility models: the (stopped)

\footnotetext{
${ }^{11}$ Note that conditions (1)-(4) in Proposition 4.3 do not depend on the correlation $\rho$, which means that the positivity of the (discounted) stock price does not depend on the correlation. Similar remarks hold for Proposition 4.4 and Proposition 4.5.

${ }^{12}$ Equivalently, we may assume that the risk-free interest rate is zero.
} 
Heston $^{13}$, the $3 / 2$, the Schöbel-Zhu and the Hull-White models. The results are summarized at the end of the section in Table 14 and Table 15.

\subsection{Stopped Heston stochastic volatility model}

Suppose that under a probability measure $P$, the (correlated) stopped Heston stochastic volatility model has the following diffusive dynamics

$$
\begin{aligned}
& d S_{t}=S_{t} \sqrt{Y_{t}} \mathbb{1}_{t \in[0, \zeta)} d W_{t}^{(1)}, \quad S_{0}=1 . \\
& d Y_{t}=\kappa\left(\theta-Y_{t}\right) \mathbb{1}_{t \in[0, \zeta)} d t+\xi \sqrt{Y_{t}} \mathbb{1}_{t \in[0, \zeta)} d W_{t}, \quad Y_{0}=x_{0}>0,
\end{aligned}
$$

with $\mathbb{E}^{P}\left[d W_{t}^{(1)} d W_{t}\right]=\rho d t,-1 \leqslant \rho \leqslant 1, \kappa>0, \theta>0, \xi>0$. The natural state space for $Y$ is $J=(\ell, r)=(0, \infty) . \zeta$ is the possible exit time of the process $Y$ from its state space $J$. The model (24) belongs to the general stochastic volatility model considered in (5) with $\mu(x)=\kappa(\theta-x)$, $\sigma(x)=\xi \sqrt{x}$, and $b(x)=\sqrt{x}$. Clearly $\sigma(x)=\xi \sqrt{x} \neq 0, x \in J, \frac{1}{\sigma^{2}(x)}=\frac{1}{\xi^{2} x} \in L_{l o c}^{1}(J), \frac{\mu(x)}{\sigma^{2}(x)}=$ $\frac{\kappa(\theta-x)}{\xi^{2} x} \in L_{l o c}^{1}(J)$, and $\frac{b^{2}(x)}{\sigma^{2}(x)}=\frac{1}{\xi^{2}} \in L_{l o c}^{1}(J)$ are satisfied. Thus, the conditions (2) and (6) are satisfied. From Proposition 2.3, under $\widetilde{P}$, the diffusion $Y$ satisfies the following SDE

$$
d Y_{t}=\widetilde{\kappa}\left(\widetilde{\theta}-Y_{t}\right) \mathbb{1}_{t \in[0, \zeta)} d t+\xi \sqrt{Y_{t}} \mathbb{1}_{t \in[0, \zeta)} d \widetilde{W}_{t}, \quad Y_{0}=x_{0}>0,
$$

where $\widetilde{\kappa}=\kappa-\rho \xi$ and $\widetilde{\theta}=\frac{\kappa \theta}{\kappa-\rho \xi}$.

For a constant $c \in J$, the scale functions of the $\operatorname{SDE}(1)$ and the $\operatorname{SDE}$ (13) are respectively

$$
\begin{aligned}
& s(x)=e^{\frac{2 \kappa c}{\xi^{2}}} c^{\frac{2 \kappa \theta}{\xi^{2}}} \int_{c}^{x} y^{-\frac{2 \kappa \theta}{\xi^{2}}} e^{\frac{2 \kappa y}{\xi^{2}}} d y=C_{1} \int_{c}^{x} y^{-\alpha} e^{\beta y} d y, \\
& \widetilde{s}(x)=e^{\frac{2 \widetilde{\kappa} c}{\xi^{2}}} c^{\frac{2 \widetilde{\kappa} \widetilde{\theta}}{\xi^{2}}} \int_{c}^{x} y^{-\frac{2 \widetilde{\kappa} \tilde{\theta}}{\xi^{2}}} e^{\frac{2 \widetilde{\kappa} y}{\xi^{2}}} d y=C_{2} \int_{c}^{x} y^{-\alpha} e^{\gamma y} d y,
\end{aligned}
$$

with $\alpha=\frac{2 \kappa \theta}{\xi^{2}}, \beta=\frac{2 \kappa}{\xi^{2}}>0, \gamma=\frac{2 \kappa}{\xi^{2}}-\frac{2 \rho}{\xi}$, and the constant terms are $C_{1}=e^{\frac{2 \kappa c}{\xi^{2}}} c^{\frac{2 \kappa \theta}{\xi^{2}}}>0$ and $C_{2}=e^{\frac{2 \kappa c}{\xi^{2}}-\frac{2 \rho c}{\xi}} c^{\frac{2 \kappa \theta}{\xi^{2}}}>0$. Under $\widetilde{P}$, we have the following test functions for $x \in \bar{J}$,

$$
\widetilde{v}(x)=\frac{2}{\xi^{2}} \int_{c}^{x} \frac{\int_{y}^{x} z^{-\alpha} e^{\gamma z} d z}{y^{1-\alpha} e^{\gamma y}} d y, \quad \widetilde{v}_{b}(x)=\frac{2}{\xi^{2}} \int_{c}^{x} \frac{\int_{y}^{x} z^{-\alpha} e^{\gamma z} d z}{y^{-\alpha} e^{\gamma y}} d y .
$$

Proposition 5.1. For ${ }^{14}$ the stopped Heston model (24), the underlying stock price $\left(S_{t}\right)_{0 \leqslant t<\infty}$ is a true P-martingale.

Proof. The proof of Proposition 5.1 is elementary and details are given in Appendix B.1. To prove it, we check the conditions of Proposition 4.1: the results are summarized in Table 5.

From Table 5 and Proposition $\left.4.1,\left(S_{t}\right)_{0 \leqslant t<\infty}\right)$ is a true $P$-martingale.

\footnotetext{
${ }^{13}$ The volatility is stopped whenever it hits the boundary 0 . When $2 \kappa \theta>\xi^{2}$ (zero is unattainable), our model coincides with the usual Heston model.

${ }^{14}$ Proposition 5.1 is consistent with Proposition 2.5, page 34 of Andersen and Piterbarg (2007), also see Remark 4.2, page 2052 of Del Baño Rollin et al. (2010).
} 


\begin{tabular}{c|c|c|c|c} 
Case & $\widetilde{v}(\ell)$ & $\widetilde{v}(r)$ & $\widetilde{v}_{b}(\ell)$ & $\widetilde{v}_{b}(r)$ \\
\hline$\alpha \geqslant 1$ & $\infty$ & $\infty$ & $\infty$ & $\infty$ \\
\hline$\alpha<1$ & $<\infty$ & $\infty$ & $<\infty$ & $\infty$ \\
\hline
\end{tabular}

Table 5: First classification table for the Heston model

Proposition 5.2. For the stopped Heston model (24), the underlying stock price $\left(S_{t}\right)_{0 \leqslant t \leqslant \infty}$ is a uniformly integrable $P$-martingale if and only if $\rho \xi \leqslant \kappa<\frac{\xi^{2}}{2 \theta}$.

Note that the Feller condition has to be violated in order to have a UI martingale.

Proof. The proof of Proposition 5.2 is elementary and details are given in Appendix B.2. To prove it, we check the conditions of Proposition 4.2: the results are summarized in Table 6.

\begin{tabular}{cc|c|c|c|c|c|c}
\multicolumn{1}{c|}{ Case } & $\widetilde{s}(\ell)$ & $\widetilde{s}(r)$ & $\widetilde{v}(\ell)$ & $\widetilde{v}(r)$ & $\widetilde{v}_{b}(\ell)$ & $\widetilde{v}_{b}(r)$ \\
\hline \multirow{4}{*}{$\alpha>1$} & $\gamma<0$ & $-\infty$ & $<\infty$ & $\infty$ & $\infty$ & $\infty$ & $\infty$ \\
\cline { 2 - 8 } & $\gamma=0$ & $-\infty$ & $<\infty$ & $\infty$ & $\infty$ & $\infty$ & $\infty$ \\
\cline { 2 - 8 } & $\gamma>0$ & $-\infty$ & $\infty$ & $\infty$ & $\infty$ & $\infty$ & $\infty$ \\
\hline \hline \multirow{3}{*}{$\alpha=1$} & $\gamma<0$ & $-\infty$ & $<\infty$ & $\infty$ & $\infty$ & $\infty$ & $\infty$ \\
\cline { 2 - 8 } & $\gamma=0$ & $-\infty$ & $\infty$ & $\infty$ & $\infty$ & $\infty$ & $\infty$ \\
\cline { 2 - 8 } & $\gamma>0$ & $-\infty$ & $\infty$ & $\infty$ & $\infty$ & $\infty$ & $\infty$ \\
\hline \hline \multirow{2}{*}{$\alpha<1$} & $\gamma<0$ & $>-\infty$ & $<\infty$ & $<\infty$ & $\infty$ & $<\infty$ & $\infty$ \\
\cline { 2 - 8 } & $\gamma=0$ & $>-\infty$ & $\infty$ & $<\infty$ & $\infty$ & $<\infty$ & $\infty$ \\
\cline { 2 - 8 } & $\gamma>0$ & $>-\infty$ & $\infty$ & $<\infty$ & $\infty$ & $<\infty$ & $\infty$ \\
\hline
\end{tabular}

Table 6: Second classification table for the Heston model

From Table 6 and Proposition 4.2, $\left(S_{t}\right)_{0 \leqslant t \leqslant \infty}$ is a uniformly integrable $P$-martingale if and only if $\alpha=\frac{2 \kappa \theta}{\xi^{2}}<1$, and $\gamma=\frac{2(\kappa-\rho \xi)}{\xi^{2}} \geqslant 0$, which is equivalent to $\rho \xi \leqslant \kappa<\frac{\xi^{2}}{2 \theta}$.

Under $P$, we have the following result on the positivity of the stock price in the stopped Heston model.

Proposition 5.3. For the stopped Heston model (24),

(1) $P\left(S_{T}>0\right)=1$ for all $T \in(0, \infty)$,

(2) $P\left(S_{\infty}>0\right)=1$ if and only if $\kappa<\frac{\xi^{2}}{2 \theta}$.

Proof. Similar to the proofs of Proposition 5.1 and Proposition 5.2 with $\gamma$ replaced by $\beta>0$ and $C_{2}$ by $C_{1}$, we obtain the classification in Table 7 .

\begin{tabular}{c|c|c|c|c|c|c} 
Case & $s(\ell)$ & $s(r)$ & $v(\ell)$ & $v(r)$ & $v_{b}(\ell)$ & $v_{b}(r)$ \\
\hline$\alpha>1$ & $-\infty$ & $\infty$ & $\infty$ & $\infty$ & $\infty$ & $\infty$ \\
\hline$\alpha=1$ & $-\infty$ & $\infty$ & $\infty$ & $\infty$ & $\infty$ & $\infty$ \\
\hline$\alpha<1$ & $>-\infty$ & $\infty$ & $<\infty$ & $\infty$ & $<\infty$ & $\infty$ \\
\hline
\end{tabular}

Table 7: Third classification table for the Heston model 
Based on Table 7, from Proposition 4.3 and Proposition 4.4, we obtain the desired results.

\section{$5.2 \quad 3 / 2$ stochastic volatility model}

Under $P$, the (correlated) $3 / 2$ stochastic volatility model has the following diffusive dynamics

$$
\begin{aligned}
& d S_{t}=S_{t} \sqrt{Y_{t}} \mathbb{1}_{t \in[0, \zeta)} d W_{t}^{(1)}, \quad S_{0}=1, \\
& d Y_{t}=\left(\omega Y_{t}-\theta Y_{t}^{2}\right) \mathbb{1}_{t \in[0, \zeta)} d t+\xi Y_{t}^{\frac{3}{2}} \mathbb{1}_{t \in[0, \zeta)} d W_{t}, \quad Y_{0}=x_{0}>0,
\end{aligned}
$$

where $\mathbb{E}^{P}\left[d W_{t}^{(1)} d W_{t}\right]=\rho d t,-1 \leqslant \rho \leqslant 1, \omega>0, \xi>0, \theta \in \mathbb{R}$. The natural state space is given by $J=(\ell, r)=(0, \infty)$. $\zeta$ is the possible exit time of the process $Y$ from its state space $J$. The model (26) belongs to the general stochastic volatility model considered in (5) with $\mu(x)=\omega x-\theta x^{2}, \sigma(x)=\xi x^{3 / 2}$, and $b(x)=\sqrt{x}$. Clearly $\sigma(x)=\xi x^{3 / 2} \neq 0, x \in J$, $\frac{1}{\sigma^{2}(x)}=\frac{1}{\xi^{2} x^{3}} \in L_{l o c}^{1}(J), \frac{\mu(x)}{\sigma^{2}(x)}=\frac{\omega-\theta x}{\xi^{2} x^{2}} \in L_{l o c}^{1}(J)$, and $\frac{b^{2}(x)}{\sigma^{2}(x)}=\frac{1}{\xi^{2} x^{2}} \in L_{l o c}^{1}(J)$ are satisfied. Thus, the conditions (2) and (6) are satisfied. From Proposition 2.3, under $\widetilde{P}$, the diffusion $Y$ satisfies the following SDE

$$
d Y_{t}=\left(\omega Y_{t}-\widetilde{\theta} Y_{t}^{2}\right) \mathbb{1}_{t \in[0, \zeta)} d t+\xi Y_{t}^{\frac{3}{2}} \mathbb{1}_{t \in[0, \zeta)} d \widetilde{W}_{t}, \quad Y_{0}=x_{0}>0,
$$

where $\widetilde{\theta}=\theta-\rho \xi$. For a constant $c \in J$, the scale functions of the SDE (1) and the SDE (13) are respectively

$$
s(x)=\frac{b}{c^{a}} \int_{c}^{x} y^{a} \exp \left(\frac{d}{y}\right) d y, \quad \widetilde{s}(x)=\frac{b}{c^{\widetilde{a}}} \int_{c}^{x} y^{\widetilde{a}} \exp \left(\frac{d}{y}\right) d y, \quad x \in \bar{J},
$$

where $a=\frac{2 \theta}{\xi^{2}}, b=\exp \left(-\frac{2 \omega}{c \xi^{2}}\right), d=\frac{2 \omega}{\xi^{2}}$ and $\widetilde{a}=a-\frac{2 \rho}{\xi}$. Since the only difference between $s(\cdot)$ and $\widetilde{s}(\cdot)$ is in the parameters $a$ and $\widetilde{a}$, the analysis under $\widetilde{P}$ is similar to the analysis under $P$, except with a change of the parameter from $a$ to $\widetilde{a}$. Thus, we only need the results under $P$. We have the following test functions

$$
\begin{aligned}
v(x) & =\frac{2}{\xi^{2}} \int_{c}^{x} \frac{1}{y^{a+3} \exp \left(\frac{d}{y}\right)}\left(\int_{y}^{x} z^{a} \exp \left(\frac{d}{z}\right) d z\right) d y, \\
v_{b}(x) & =\frac{2}{\xi^{2}} \int_{c}^{x} \frac{1}{y^{a+2} \exp \left(\frac{d}{y}\right)}\left(\int_{y}^{x} z^{a} \exp \left(\frac{d}{z}\right) d z\right) d y .
\end{aligned}
$$

Lemma 5.1. With $\omega>0$, the following properties are satisfied.

$$
\begin{array}{ll|ll}
\text { (i) } & a<-1 \Longleftrightarrow v(r)<\infty, & (v) & \widetilde{a}<-1 \Longleftrightarrow \widetilde{v}(r)<\infty . \\
\text { (ii) } \quad \forall a \in \mathbb{R}, \quad v_{b}(r)=\infty, & (v i) & \forall \widetilde{a} \in \mathbb{R}, \quad \widetilde{v}_{b}(r)=\infty . \\
\text { (iii) } \quad \forall a \in \mathbb{R}, \quad v(\ell)=\infty, & (v i i) & \forall \widetilde{a} \in \mathbb{R}, \quad \widetilde{v}(\ell)=\infty . \\
\text { (iv) } \quad \forall a \in \mathbb{R}, \quad v_{b}(\ell)=\infty, & (\text { viii }) & \forall \widetilde{a} \in \mathbb{R}, \quad \widetilde{v}_{b}(\ell)=\infty .
\end{array}
$$

Proof. Details of the derivations can be found in Appendix B.3.

Proposition 5.4. For ${ }^{15}$ the $3 / 2$ model (26), the underlying stock price $\left(S_{t}\right)_{0 \leqslant t<\infty}$ is a true Pmartingale if and only if $\xi^{2}-2 \rho \xi+2 \theta \geqslant 0$.

\footnotetext{
${ }^{15}$ Theorem 3, page 110 of Carr and Sun (2007) proves sufficiency. See also Lewis (2000).
} 
Proof. From Lemma 5.1 and Proposition 4.1, $\left(S_{t}\right)_{0 \leqslant t<\infty}$ is a true P-martingale if and only if $\widetilde{a} \geqslant-1$, which is equivalent to $\xi^{2}-2 \rho \xi+2 \theta \geqslant 0$ after some simplifications.

Proposition 5.5. For the $3 / 2$ model (26), the underlying stock price $\left(S_{t}\right)_{0 \leqslant t \leqslant \infty}$ is not a uniformly integrable P-martingale.

Proof. From Lemma 5.1, for all $\widetilde{a} \in \mathbb{R}, \widetilde{v}_{b}(r)=\infty$ and $\widetilde{v}_{b}(l)=\infty$ hold. From Proposition 4.2, $\left(S_{t}\right)_{0 \leqslant t \leqslant \infty}$ is not a uniformly integrable $P$-martingale.

Under $P$, we have the following result on the positivity of the stock price in the $3 / 2$ model.

Proposition 5.6. For the $3 / 2$ model (26),

(1) $P\left(S_{T}>0\right)=1$ for all $T \in(0, \infty)$ if and only if $\xi^{2}+2 \theta \geqslant 0$,

(2) $P\left(S_{\infty}>0\right)<1$.

Proof. Similar to the proofs of Proposition 5.4 and Proposition 5.5 with $\widetilde{a}$ replaced by $a$, we obtain the classification in Table 8. Based on Table 8, Proposition 4.3 and Proposition 4.4, we

\begin{tabular}{c|c|c|c|c} 
Case & $v(\ell)$ & $v(r)$ & $v_{b}(\ell)$ & $v_{b}(r)$ \\
\hline$a<-1$ & $\infty$ & $<\infty$ & $\infty$ & $\infty$ \\
\hline$a \geqslant-1$ & $\infty$ & $\infty$ & $\infty$ & $\infty$ \\
\hline
\end{tabular}

Table 8: Classification table for the $3 / 2$ model

have the desired results. Note that $a \geqslant-1$ is equivalent to $\xi^{2}+2 \theta \geqslant 0$.

\subsection{Schöbel-Zhu stochastic volatility model}

Under $P$, the correlated Schöbel-Zhu stochastic volatility model ${ }^{16}$ (see Schöbel and Zhu (1999)) can be described by the following diffusive dynamics

$$
\begin{aligned}
& d S_{t}=S_{t} Y_{t} \mathbb{1}_{t \in[0, \zeta)} d W_{t}^{(1)}, \quad S_{0}=1, \\
& d Y_{t}=\kappa\left(\theta-Y_{t}\right) \mathbb{1}_{t \in[0, \zeta)} d t+\gamma \mathbb{1}_{t \in[0, \zeta)} d W_{t}, \quad Y_{0}=x_{0},
\end{aligned}
$$

where $\mathbb{E}\left[d W_{t}^{(1)} d W_{t}\right]=\rho d t,-1 \leqslant \rho \leqslant 1, \kappa>0, \theta>0, \gamma>0$. The process $Y$ is an OrnsteinUhlenbeck process, and this implies that its natural state space is $J=(\ell, r)=(-\infty, \infty)$. $\zeta$ is the possible exit time of the process $Y$ from its state space $J$. The model (30) belongs to the general stochastic volatility model considered in (5) with $\mu(x)=\kappa(\theta-x), \sigma(x)=\gamma$, and $b(x)=x$. Clearly $\sigma(x)=\gamma \neq 0, x \in J$, then $\frac{1}{\sigma(x)^{2}}=\frac{1}{\gamma^{2}} \in L_{l o c}^{1}(J), \frac{\mu(x)}{\sigma(x)^{2}}=\frac{\kappa(\theta-x)}{\gamma^{2}} \in L_{l o c}^{1}(J)$, and $\frac{b^{2}(x)}{\sigma^{2}(x)}=\frac{x^{2}}{\gamma^{2}} \in L_{l o c}^{1}(J)$ are satisfied. Thus, the conditions (2) and (6) are satisfied.

From Proposition 2.3, under $\widetilde{P}$, the diffusion $Y$ satisfies the following SDE

$$
d Y_{t}=\left(\kappa \theta-(\kappa-\rho \gamma) Y_{t}\right) \mathbb{1}_{t \in[0, \zeta)} d t+\gamma \mathbb{1}_{t \in[0, \zeta)} d \widetilde{W}_{t}, \quad Y_{0}=x_{0} .
$$

\footnotetext{
${ }^{16}$ It is the correlated version of the Stein-Stein (1991) model. In Rheinländer (2005), the minimal entropy martingale measure is studied in detail for this model, and its Proposition 3.1 gives a necessary and sufficient condition such that the associated stochastic exponential is a true martingale. Here we provide deterministic criteria.
} 
For a positive constant $c \in J$, denote $\alpha=\kappa-\rho \gamma$, and compute the scale functions respectively of the SDE (1) and the SDE (13)

$$
\begin{aligned}
& s(x)=\int_{c}^{x} e^{\frac{\kappa(y-\theta)^{2}-\kappa(c-\theta)^{2}}{\gamma^{2}}} d y=C_{1} \int_{c}^{x} e^{\frac{\kappa(y-\theta)^{2}}{\gamma^{2}}} d y, \\
& \widetilde{s}(x)=\int_{c}^{x} e^{\frac{\alpha y^{2}-2 \kappa \theta y+2 \kappa \theta c-\alpha c^{2}}{\gamma^{2}}} d y= \begin{cases}C_{2} \int_{c}^{x} e^{\frac{\alpha\left(y-\frac{\kappa \theta}{\alpha}\right)^{2}}{\gamma^{2}}} d y, & \text { if } \alpha \neq 0, \\
C_{3}\left(e^{-\frac{2 \kappa \theta c}{\gamma^{2}}}-e^{-\frac{2 \kappa \theta}{\gamma^{2}} x}\right), & \text { if } \alpha=0,\end{cases}
\end{aligned}
$$

with constants $C_{1}=e^{-\kappa(c-\theta)^{2} / \gamma^{2}}>0, C_{2}=e^{\left(-\kappa^{2} \theta^{2} / \alpha+2 \kappa \theta c-\alpha c^{2}\right) / \gamma^{2}}>0$ for $\alpha \neq 0$, and the constant $C_{3}=e^{2 \kappa \theta c / \gamma^{2}} \frac{\gamma^{2}}{2 \kappa \theta}>0$ for $\alpha=0$. Since $\kappa>0$ by assumption, $e^{\frac{\kappa(y-\theta)^{2}}{\gamma^{2}}} \geqslant 1$ for any $y \in[c, x]$, with $c \in J, x \in \bar{J}$, then $s(r)=s(\infty)=\infty$ always holds, and consequently $v(r)=v(\infty)=\infty$.

Proposition 5.7. For the Schöbel-Zhu model (30), the underlying stock price $\left(S_{t}\right)_{0 \leqslant t<\infty}$ is a true P-martingale.

Proof. We now check the conditions in Proposition 4.1. For the case of the right endpoint $r$, depending on the sign of $\alpha=\kappa-\rho \gamma$, we obtain the following classification

$$
\widetilde{s}(\infty) \begin{cases}<\infty, & \text { if } \alpha \leqslant 0 \\ =\infty, & \text { if } \alpha>0\end{cases}
$$

Details can be found in Appendix B.4. Above all, we can summarize the results in Table 9.

\begin{tabular}{c|c|c|c|c|c|c} 
Case & $\widetilde{s}(\ell)$ & $\widetilde{s}(r)$ & $\widetilde{v}(\ell)$ & $\widetilde{v}(r)$ & $\widetilde{v}_{b}(\ell)$ & $\widetilde{v}_{b}(r)$ \\
\hline$\alpha \leqslant 0$ & $>-\infty$ & $<\infty$ & $<\infty$ & $\infty$ & $<\infty$ & $\infty$ \\
\hline$\alpha>0$ & $>-\infty$ & $\infty$ & $<\infty$ & $\infty$ & $<\infty$ & $\infty$ \\
\hline
\end{tabular}

Table 9: First classification table for the Schöbel-Zhu model

From Proposition $4.1(3),\left(S_{t}\right)_{0 \leqslant t<\infty}$ is a true $P$-martingale.

Proposition 5.8. For the Schöbel-Zhu model (30), the underlying stock price $\left(S_{t}\right)_{0 \leqslant t \leqslant \infty}$ is a uniformly integrable $P$-martingale if and only if $\kappa>\rho \gamma$.

Proof. From Table 9 and Proposition 4.2, it follows that $\left(S_{t}\right)_{0 \leqslant t \leqslant \infty}$ is a uniformly integrable $P$-martingale if and only if $\alpha>0$, or equivalently $\kappa>\rho \gamma$.

Under $P$, we obtain the following result on the positivity of the stock price in the Schöbel-Zhu model.

Proposition 5.9. For the Schöbel-Zhu model (30),

(1) $P\left(S_{T}>0\right)=1$ for all $T \in(0, \infty)$,

(2) $P\left(S_{\infty}>0\right)=1$.

Proof. Similar to the proofs of Proposition 5.7 and Proposition 5.8 with $\alpha$ replaced by $\kappa>0$, we obtain the classification given in Table 10. 


\begin{tabular}{l|l|l|l|l|l|l} 
Case & $s(\ell)$ & $s(r)$ & $v(\ell)$ & $v(r)$ & $v_{b}(\ell)$ & $v_{b}(r)$ \\
\hline$\alpha>0$ & $>-\infty$ & $\infty$ & $<\infty$ & $\infty$ & $<\infty$ & $\infty$ \\
\hline
\end{tabular}

Table 10: Second classification table for the Schöbel-Zhu model

\subsection{Hull-White stochastic volatility model}

Under $P$, the correlated Hull-White stochastic volatility model (see Hull and White (1987)) can be described by the following diffusive dynamics

$$
\begin{aligned}
& d S_{t}=S_{t} \sqrt{Y_{t}} \mathbb{1}_{t \in[0, \zeta)} d W_{t}^{(1)}, \quad S_{0}=1, \\
& d Y_{t}=\mu Y_{t} \mathbb{1}_{t \in[0, \zeta)} d t+\sigma Y_{t} \mathbb{1}_{t \in[0, \zeta)} d W_{t}, \quad Y_{0}=x_{0}>0,
\end{aligned}
$$

where $\mathbb{E}\left[d W_{t}^{(1)} d W_{t}\right]=\rho d t,-1 \leqslant \rho \leqslant 1, \mu>0$, and $\sigma>0$. The process $Y$ is a geometric Brownian motion process, and this implies that its natural state space is $J=(\ell, r)=(0, \infty)$. $\zeta$ is the possible exit time of the process $Y$ from its state space $J$. The model (31) belongs to the general stochastic volatility model considered in (5) with $\mu(x)=\mu x, \sigma(x)=\sigma x$, and $b(x)=\sqrt{x}$. Clearly $\sigma(x)=\sigma x \neq 0, x \in J, \frac{1}{\sigma(x)^{2}}=\frac{1}{\sigma^{2} x^{2}} \in L_{l o c}^{1}(J), \frac{\mu(x)}{\sigma(x)^{2}}=\frac{\mu}{\sigma^{2} x} \in L_{l o c}^{1}(J)$, and $\frac{b^{2}(x)}{\sigma^{2}(x)}=\frac{1}{\sigma^{2} x} \in L_{l o c}^{1}(J)$ are satisfied. Thus, the conditions (2) and (6) are satisfied.

From Proposition 2.3, under $\widetilde{P}$, the diffusion $Y$ satisfies the following SDE

$$
d Y_{t}=\left(\mu Y_{t}+\rho \sigma Y_{t}^{\frac{3}{2}}\right) \mathbb{1}_{t \in[0, \zeta)} d t+\sigma Y_{t} \mathbb{1}_{t \in[0, \zeta)} d \widetilde{W}_{t}, \quad Y_{0}=x_{0}>0,
$$
(13)

Denote $\alpha=\frac{4 \mu}{\sigma^{2}}-1$ and $\gamma=\frac{4 \rho}{\sigma}$. For a constant $c \in J$, compute the scale functions of the SDE

$$
\widetilde{s}(x)=\int_{c}^{x} e^{-\int_{c}^{y} \frac{2 \mu u+2 \rho \sigma u^{3 / 2}}{\sigma^{2} u^{2}} d u} d y=C_{1} \int_{c}^{x} y^{-\frac{\alpha+1}{2}} e^{-\gamma \sqrt{y}} d y, \quad x \in \bar{J},
$$

where $C_{1}=c^{\frac{2 \mu}{\sigma^{2}}} e^{\frac{4 \rho}{\sigma} \sqrt{c}}$ is a positive constant. From the definition in (17) and the scale function in (33)

$$
\widetilde{v}(x)=\int_{c}^{x} \frac{2(\widetilde{s}(x)-\widetilde{s}(y))}{\widetilde{s}^{\prime}(y) \widetilde{\sigma}^{2}(y)} d y=\frac{2}{\sigma^{2}} \int_{c}^{x} y^{\frac{\alpha-3}{2}} e^{\gamma \sqrt{y}}\left(\int_{y}^{x} z^{-\frac{\alpha+1}{2}} e^{-\gamma \sqrt{z}} d z\right) d y,
$$

and

$$
\widetilde{v}_{b}(x)=\frac{2}{\sigma^{2}} \int_{c}^{x} y^{\frac{\alpha-1}{2}} e^{\gamma \sqrt{y}}\left(\int_{y}^{x} z^{-\frac{\alpha+1}{2}} e^{-\gamma \sqrt{z}} d z\right) d y
$$

Proposition 5.10. For ${ }^{17}$ the Hull-White model (31), the underlying stock price $\left(S_{t}\right)_{0 \leqslant t<\infty}$ is a true $P$-martingale if and only if $\rho \leqslant 0$.

\footnotetext{
${ }^{17}$ Proposition 5.10 is consistent with Theorem 1 of Jourdain (2004), and Proposition 2.5., page 34 of Andersen and Piterbarg (2007).
} 
Proof. We distinguish 3 situations: (I): $\mu>\frac{1}{2} \sigma^{2}$, (II): $\mu=\frac{1}{2} \sigma^{2}$ and (III): $\mu<\frac{1}{2} \sigma^{2}$. Results are summarized in Table 11. Details can be found in Appendix B.5. The results in Table 11, combined with Proposition 4.1 allow us to conclude if $\left(S_{t}\right)_{0 \leqslant t \leqslant T}, T \in(0, \infty)$ is a true $P$-martingale. For $2 \mu / \sigma^{2}>1(\alpha>1),\left(S_{t}\right)_{0 \leqslant t \leqslant T}, T \in(0, \infty)$ is a true $P$-martingale if and only if $\widetilde{v}(r)=\infty$. This is equivalent to $\gamma \leqslant 0$, and further equivalent to $\rho \leqslant 0$ from the definition of $\gamma$. When $2 \mu / \sigma^{2}=1$ $(\alpha=1),\left(S_{t}\right)_{0 \leqslant t \leqslant T}, T \in(0, \infty)$ is a true $P$-martingale if and only if $\widetilde{v}(r)=\infty$, equivalently $\gamma \leqslant 0$, that is $\rho \leqslant 0$. When $2 \mu / \sigma^{2}<1(\alpha<1),\left(S_{t}\right)_{0 \leqslant t \leqslant T}, T \in(0, \infty)$ is a true $P$-martingale if and only if $\widetilde{v}(r)=\infty$, equivalently $\gamma \leqslant 0$, that is $\rho \leqslant 0$.

\begin{tabular}{cc|c|c|c|c} 
Case & & $\widetilde{v}(\ell)$ & $\widetilde{v}(r)$ & $\widetilde{v}_{b}(\ell)$ & $\widetilde{v}_{b}(r)$ \\
\hline \multirow{2}{*}{$(\mathrm{I}) \mu>\frac{\sigma^{2}}{2}$} & $\gamma \leqslant 0$ & $\infty$ & $\infty$ & $\infty$ & $\infty$ \\
\cline { 2 - 6 } & $\gamma>0$ & $\infty$ & $<\infty$ & $\infty$ & $\infty$ \\
\hline \hline \multirow{2}{*}{$(\mathrm{II}) \mu=\frac{\sigma^{2}}{2}$} & $\gamma \leqslant 0$ & $\infty$ & $\infty$ & $\infty$ & $\infty$ \\
\cline { 2 - 6 } & $\gamma>0$ & $\infty$ & $<\infty$ & $\infty$ & $\infty$ \\
\hline \hline \multirow{2}{*}{ (III) $\mu<\frac{\sigma^{2}}{2}$} & $\gamma \leqslant 0$ & $\infty$ & $\infty$ & $<\infty$ & $\infty$ \\
\cline { 2 - 6 } & $\gamma>0$ & $\infty$ & $<\infty$ & $<\infty$ & $\infty$ \\
\hline
\end{tabular}

Table 11: First classification table for the Hull-White model

Proposition 5.11. For the Hull-White model (31), the underlying stock price $\left(S_{t}\right)_{0 \leqslant t \leqslant \infty}$ is a uniformly integrable $P$-martingale if and only if $\mu<\frac{1}{2} \sigma^{2}$ and $\rho \leqslant 0$.

Proof. The proof of Proposition 5.11 requires the same 3 cases as Proposition 5.10. Results are summarized in Table 12. Details can be found in Appendix B.6.

\begin{tabular}{|c|c|c|c|c|c|c|c|}
\hline Case & & $\widetilde{s}(\ell)$ & $\widetilde{s}(r)$ & $\widetilde{v}(\ell)$ & $\widetilde{v}(r)$ & $\widetilde{v}_{b}(\ell)$ & $\widetilde{v}_{b}(r)$ \\
\hline \multirow{3}{*}{ (I) $\mu>\frac{\sigma^{2}}{2}$} & $\gamma>0$ & $-\infty$ & $<\infty$ & $\infty$ & $<\infty$ & $\infty$ & $\infty$ \\
\hline & $\gamma=0$ & $-\infty$ & $<\infty$ & $\infty$ & $\infty$ & $\infty$ & $\infty$ \\
\hline & $\gamma<0$ & $-\infty$ & $\infty$ & $\infty$ & $\infty$ & $\infty$ & $\infty$ \\
\hline \multirow{3}{*}{ (II) $\mu=\frac{\sigma^{2}}{2}$} & $\overline{\gamma \gamma>0}$ & $-\infty$ & $\langle<$ & $\infty$ & $<\infty$ & $\infty$ & 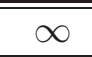 \\
\hline & $\gamma=0$ & $-\infty$ & $\infty$ & $\infty$ & $\infty$ & $\infty$ & $\infty$ \\
\hline & $\gamma<0$ & $-\infty$ & $\infty$ & $\infty$ & $\infty$ & $\infty$ & $\infty$ \\
\hline \multirow{3}{*}{ (III) $\mu<\frac{\sigma^{2}}{2}$} & $\gamma>0$ & $>-\infty$ & $<\infty$ & $\infty$ & $<\infty$ & $<\infty$ & $\infty$ \\
\hline & $\gamma=0$ & $>-\infty$ & $\infty$ & $\infty$ & $\infty$ & $<\infty$ & $\infty$ \\
\hline & $\gamma<0$ & $>-\infty$ & $\infty$ & $\infty$ & $\infty$ & $<\infty$ & $\infty$ \\
\hline
\end{tabular}

Table 12: Second classification table for the Hull-White model

Under $P$, we have the following result on the positivity of the stock price in the Hull-White model.

Proposition 5.12. For the Hull-White model (31),

(1) $P\left(S_{T}>0\right)=1$ for all $T \in(0, \infty)$,

(2) $P\left(S_{\infty}>0\right)=1$ if and only if $\frac{2 \mu}{\sigma^{2}}<1$. 
Proof. Similar to the proofs of Proposition 5.10 and Proposition 5.11 with $\gamma=0$, we have the classification in Table 13.

\begin{tabular}{c|c|c|c|c|c|c} 
Case & $s(\ell)$ & $s(r)$ & $v(\ell)$ & $v(r)$ & $v_{b}(\ell)$ & $v_{b}(r)$ \\
\hline (I) $\mu>\frac{\sigma^{2}}{2}$ & $-\infty$ & $<\infty$ & $\infty$ & $\infty$ & $\infty$ & $\infty$ \\
\hline (II) $\mu=\frac{\sigma^{2}}{2}$ & $-\infty$ & $\infty$ & $\infty$ & $\infty$ & $\infty$ & $\infty$ \\
\hline (III) $\mu<\frac{\sigma^{2}}{2}$ & $>-\infty$ & $\infty$ & $\infty$ & $\infty$ & $<\infty$ & $\infty$ \\
\hline
\end{tabular}

Table 13: Third classification table for the Hull-White model

From Table 13, Proposition 4.3 and Proposition 4.4, we obtain the desired results.

\subsection{Summary of the Examples}

Table 14 and Table 15 summarize the results obtained throughout Section 5 . In all cases, we study the "stopped" price process as we assume that there are two absorbing barriers at $\ell$ and $r$. Conditions for uniformly integrable martingales are stronger than those for a true martingale on $(0, \infty)$. Similar remarks hold for the positivity of $S_{T}$ and $S_{\infty}$, where $0<T<\infty$.

\begin{tabular}{c|c|c} 
Model & True martingale on $(0, \infty)$ & UI martingale on $[0, \infty]$ \\
\hline Heston Model $(24)$ & under Feller condition & never under Feller condition \\
& $\kappa>\frac{\xi^{2}}{2 \theta}$ & $\rho \xi \leqslant \kappa<\frac{\xi^{2}}{2 \theta}$ \\
\hline $3 / 2$ Model $(26)$ & $\xi^{2}+2 \theta \geqslant \max (0,2 \rho \xi)$ & Never \\
\hline Schöbel-Zhu Model $(30)$ & Always & when $\kappa>\rho \gamma$ \\
\hline Hull White Model $(31)$ & $\rho \leqslant 0$ & $\mu<\frac{\sigma^{2}}{2}$ and $\rho \leqslant 0$ \\
\hline
\end{tabular}

Table 14: Summary of conditions for (uniformly integrable) martingales.

\begin{tabular}{c|c|c} 
Model & $S_{T}$ positive $P$-a.s. & $S_{\infty}$ positive $P$-a.s. \\
\hline Heston Model $(24)$ & Always & $\begin{array}{c}\text { never under Feller condition } \\
\kappa<\frac{\xi^{2}}{2 \theta}\end{array}$ \\
\hline 3/2 Model $(26)$ & $\xi^{2}+2 \theta \geqslant 0$ & Never \\
\hline Schöbel-Zhu Model (30) & Always & Always \\
\hline Hull White Model $(31)$ & Always & $\mu<\frac{\sigma^{2}}{2}$ \\
\hline
\end{tabular}

Table 15: Summary of conditions for positivity of stock prices.

\section{Concluding Remarks}

This paper generalizes some results of Mijatović and Urusov (2012b, 2012c) concerning the (uniformly integrable) martingale property of the asset price from the case $\rho=1$ to the case $-1 \leqslant \rho \leqslant 1$, and provides new direct proofs without using the concept of "separating times". We 
also obtain deterministic criteria for the convergence or divergence of both perpetual and capped integral functionals of time-homogeneous diffusions. Explicit deterministic criteria for checking the (uniformly integrable) martingale properties for four stochastic volatility models are provided. Future research directions include finding necessary and sufficient deterministic conditions for the martingale property of time-changed Lévy processes with non-zero correlation (Carr and Wu (2004)), of which the time-homogeneous stochastic volatility models considered in this paper are special cases.

\section{References}

Andersen, L., And V. Piterbarg (2007): "Moment explosions in stochastic volatility models," Finance and Stochastics, 11, 29-50.

Bayraktar, E., C. Kardaras, And H. Xing (2012): "Valuation equations for stochastic volatility models," SIAM Journal in Financial Mathematics, 3, 351-373.

Blanchet, J., AND J. Ruf (2012): "A weak convergence criterion constructing changes of measure," working paper, available at Arxiv: http://arxiv. org/abs/1208. 2606.

Blei, S., And H. Engelbert (2009): "On exponential local martingales associated with strong Markov continuous local martingales," Stochastic Processes and their Applications, 119, 28592880.

Carr, P., T. Fisher, and J. Ruf (2014): "On the hedging of options on exploding exchange rates," Finance and Stochastics, 18, 115-144.

CARr, P., AND J. Sun (2007): "A new approach of option pricing under stochastic volatility," Review of Derivatives Research, 10, 87-150.

Carr, P., And L. Wu (2004): "Time-changed Lévy processes and option pricing," Journal of Financial Economics, 71, 113-141.

Cherny, A., and A. Shiryaev (2001): "On criteria for the uniform integrability of Brownian stochastic exponentials," In: Optimal Control and Partial Differential Equations. In honor of Alain Bensoussan's 60th birthday, pp. 80-92.

Cherny, A., And M. Urusov (2004): "Separating times for measures on filtered spaces," Theory of Probability and Applications, 48, 337-347.

(2006): "On the absolute continuity and singularity of measures on filtered spaces: separating times," From Stochastic Calculus to Mathematical Finance, The Shiryaev Festschrift, Eds. Yu. Kabanov, R.Liptser, J.Stoyanov. Springer, Berlin, pp. 125-168.

CuI, Z. (2014): "A new proof of an Engelbert-Schmidt type zero-one law for time-homogeneous diffusions," Statistics and Probability Letters, 89, 118-123.

Del Baño Rollin, S., A. Ferreiro-Castilla, and F. Utzet (2010): "On the density of logspot in Heston volatility model," Stochastic Processes and their Applications, 120, 2037-2062. 
Delbaen, F., and H. Shirakawa (2002): "No arbitrage condition for positive diffusion price processes," Asia-Pacific Financial Markets, 9, 159-168.

Doss, H., and E. Lenglart (1978): "Sur l'existence, l'unicité et le comportement asymptotique des solutions déquations différentielles stochastiques," Annales de l'institut Henri Poincaré: (B) Probabilités et Statistiques, 14(2), 189-214.

Engelbert, H., And W. Schmidt (1981): "On the behavior of certain functionals of the Wiener process and applications to stochastic differential equations," Stochastic Differential Systems (Visegrád, 1980), volume 36 of Lecture Notes in Control and Information Science, pp. 47-55.

— (1984): "On exponential local martingales connected with diffusion processes," Mathematische Nachrichten, 119, 97-115.

Engelbert, H., And G. TitTel (2002): "Integral functionals of strong markov continuous local martingales," Stochastics Monographs, 12, 111-132.

FöllmeR, H. (1972): "The exit measure of a supermartingale," Zeitschrift fur Wahrscheinlichkeitstheorie und Verwandte Gebiete, 21, 154-166.

Gikhman, I., And A. Skorohod (1972): "Stochastic differential equations," Springer.

Girsanov, I. (1960): "On transforming a certain class of stochastic processes by absolutely continuous substitution of measures," Theory of Probability and its Applications, 5(3), 285301.

Heston, S. (1993): "A Closed-form Solution for Options with Stochastic Volatility with Applications to Bond and Currency Options," The Review of Financial Studies, 6, 327-343.

Hull, J., And A. White (1987): "The Pricing of Options on Assets with Stochastic Volatilities," Journal of Finance, 42(2), 281-300.

Hulley, H., and E. Platen (2011): "A visual criterion for identifying Itō diffusions as martingales or strict local martingales," Seminar on Stochastic Analysis, Random Fields and Applications VI, Birkhäuser, pp. 147-157.

Jourdain, B. (2004): "Loss of martingality in asset price models with lognormal stochastic volatility," Preprint CERMICS 2004-267, available at http://cermics. enpc. fr/reports/CERMICS-2004/CERMICS-2004-267. pdf.

Kallsen, J., And J. Muhle-Karbe (2010): "Exponentially affine martingales, affine measure changes and exponential moments of affine processes," Stochastic Processes and their Applications, 120(2), 163-181.

Kallsen, J., and A. Shiryaev (2002): "The cumulant process and the Esscher's change of measure," Finance and Stochastics, 6(4), 397-428.

Karatzas, I., And J. RuF (2013): "Distribution of the time of explosions for one-dimensional diffusions," working paper, available at Arxiv: http://arxiv. org/abs/1303. 5899.

Karatzas, I., And S. Shreve (1991): Brownian motion and stochastic calculus. Springer. 
Kazamaki, N. (1977): "On a problem of Girsanov," The Tohoku Mathematical Journal, 29, $597-600$.

Khoshnevisan, D., P. Salminen, and M. Yor (2006): "A note on a.s. finiteness of perpetual integral functionals of diffusions," Electronic Communications in Probability, 11, 108-117.

Kotani, S. (2006): "On a condition that one-dimensional diffusion processes are martingales," Séminaire de Probabilités XXXIX, Lecture Notes in Mathematics, Srpinger, Berlin, 1874, 149156.

Kramkov, D., and A. Shiryaev (1998): "Sufficient conditions for the uniform integrability of exponential martingales," Progress in Mathematics, 168, 289-295.

LEwIS, A. (2000): "Option valuation under stochastic volatility with Mathematica code," Newport Beach, CA: Financial Press.

Lions, P., And M. Musiela (2007): "Correlations and bounds for stochastic volatility models," Annals of Institute of Henri Poincare, 24, 1-16.

Liptser, R., And A. Shiryaev (1972): "On the absolute continuity of measures corresponding to processes of diffusion type relative to a Wiener measure," Mathematics of the USSR-Izvestiya, $6,839-882$.

Mayerhofer, E., J. Muhle-Karbe, and A. Smirnov (2011): "A characterization of the martingale property of exponentially affine processes," Stochastic Processes and their Applications, 121(3), 568-582.

Mijatović, A., N. Novak, And M. Urusov (2012): "Martingale property of generalized stochastic exponentials," Séminaire de Probabilités XLIV, Lecture Notes in Mathematics, Srpinger, Berlin, 2046, 41-59.

Mijatović, A., And M. URusov (2012a): "Convergence of integral functionals of onedimensional diffusions," Electronic Communications in Probability, 17(61), 1-13.

(2012b): "Deterministic criteria for the absence of arbitrage in one-dimensional diffusion models," Finance and Stochastics, 16, 225-247.

(2012c): "On the martingale property of certain local martingales," Probability Theory and Related Fields, 152, 1-30.

Novikov, A. (1972): "On an identity for stochastic integrals," Theory of Probability and its Applications, 17(4), 717-720.

Parthasarathy, K. (1967): Probability measures on metric spaces. Academic Press.

Revuz, D., And M. Yor (1999): "Continuous martingales and Brownian Motions, 3rd edition," Grundlehren der mathematischen Wissenschaften 293, Springer.

Rheinländer, T. (2005): "An entropy approach to the Stein and Stein model with correlation," Finance and Stochastics, 9(3), 399-413. 
- (2010): "Stochastic Exponentials," Encyclopedia of Quantitative Finance, Edited by Rama Cont.

Ruf, J. (2013a): "The martingale property in the context of stochastic differential equations," working paper.

(2013b): "A new proof for the conditions of Novikov and Kazamaki," Stochastic Processes and their Applications, 123, 404-421.

Salminen, P., And M. Yor (2006): "Properties of perpetual integral functionals of Brownian motion with drift," Ann. I.H.Poincare, 41(3), 335-347.

SchÖBel, R., AND J. ZHu (1999): "Stochastic volatility with an Ornstein-Uhlenbeck process: An extension," European Finance Review, 3(1), 23-46.

Sin, C. (1998): "Complications with stochastic volatility models," Advances in Applied Probability, 30(1), 256-268.

Stein, E., And J. Stein (1991): "Stock price distributions with stochastic volatility: an analytical approach," Review of Financial Studies, 4(4), 727-752.

Stummer, W. (1993): "The Novikov and entropy conditions of multidimensional diffusion processes with singular drift," Probability Theory and Related Fields, 97(4), 515-542. 


\section{A Technical Conditions on the Probability space and Filtration}

Throughout the paper we assume a space accommodating all four processes $\left(Y, Z, W, W^{(1)}\right)$ in (5). This is described below, following closely the presentation in Appendix B of Carr, Fisher and Ruf (2014). For a fixed time horizon $T \in(0, \infty]$, we require a stochastic basis $\left(\Omega, \mathcal{F}_{T},\left\{\mathcal{F}_{t}\right\}_{t \in[0, T]}, P\right)$ with a right-continuous filtration $\left\{\mathcal{F}_{t}\right\}_{t \in[0, T]}$. As in Carr, Fisher and Ruf (2014) and Föllmer (1972), page 156, for any stopping time $\tau$, we define $\mathcal{F}_{\tau}:=\left\{A \in \mathcal{F}_{T} \mid A \cap\{\tau \leqslant\right.$ $t\} \in \mathcal{F}_{t}$ for all $\left.t \in[0, T]\right\}$ and $\mathcal{F}_{\tau-}:=\sigma\left(\left\{A \cap\{\tau>t\} \in \mathcal{F}_{T} \mid A \in \mathcal{F}_{t}\right.\right.$ for some $\left.\left.t \in[0, T] \cup \mathcal{F}_{0}\right\}\right)$. In general, non-negative random variables are permitted to take values in the set $[0, \infty]$ and stopping times $\tau$ are permitted to take values in the set $[0, \infty] \cup \mathcal{T}$ for some transfinite time $\mathcal{T}>T$. In special cases we may restrict the range of stopping times.

Let $\Omega_{1}$ denote the space of continuous paths $\omega_{1}:[0, \infty) \rightarrow \bar{J}$ with $\omega_{1}(0) \in J$. Define $\zeta\left(\omega_{1}\right):=\inf \left\{t \in[0, T]: \omega_{1}(t) \notin J\right\}$ with the convention $\inf \emptyset=\mathcal{T}$. Assume that $\omega_{1}$ stays at either $\ell$ or $r$ once it hits it, i.e. that $\omega_{1}(\zeta+s)=\omega_{1}(\zeta)$ for all $s>0$ on the set $\{\zeta<\infty\}$. Let $\Omega_{2}$ denote the space of continuous ${ }^{18}$ paths $\omega_{2}:[0, \infty) \rightarrow[0, \infty]$ with $\omega_{2}(0)=1$. As in Carr, Fisher and Ruf (2014), define for all $i \in \mathbb{N}, R_{i}:=\inf \left\{t \in[0, T]: \omega_{2}(t)>i\right\}$, and $S_{i}:=\inf \left\{t \in[0, T]: \omega_{2}(t)<\frac{1}{i}\right\}$. Then $R:=\lim _{i \uparrow \infty} R_{i}, S:=\lim _{i \uparrow \infty} S_{i}$ are respectively the first hitting time of infinity and zero by $\omega_{2}$, with the convention $\inf \emptyset=\mathcal{T}$. Assume that $\omega_{2}(R+s)=\omega_{2}(R)$ for all $s>0$ on $\{R<\infty\}$ and similarly $\omega_{2}(S+s)=\omega_{2}(S)$ for all $s>0$ on $\{S<\infty\}$, so that $\omega_{2}$ stays at zero or infinity once it hits it. Let $\Omega_{3}$ denote the space of continuous paths $\omega_{3}:[0, \infty) \rightarrow \mathbb{R}$ with $\omega_{3}(0)=0$. Similarly let $\Omega_{4}$ denote the space of continuous paths $\omega_{4}:[0, \infty) \rightarrow \mathbb{R}$ with $\omega_{4}(0)=0$.

Denote $\Omega=\prod_{i=1}^{4} \Omega_{i}$ and $\omega=\left(\omega_{1}, \omega_{2}, \omega_{3}, \omega_{4}\right)$. As in Appendix B of Carr, Fisher and Ruf (2014), we require that $\left\{\mathcal{F}_{R_{i}-}\right\}_{i \in \mathbb{N}}$ is a standard system, see Remark 6.1.1 of Föllmer (1972), so that in the proof of Proposition 2.1, the Extension Theorem V4.1 of Parthasarathy (1967) can be applied, and any probability measure on $\mathcal{F}_{R-}$ has a (possibly non-unique) extension to a probability measure on $\mathcal{F}_{T}$. Such a canonical filtration can be constructed as in Appendix B of Carr, Fisher and Ruf (2014).

Given the canonical space $\left(\Omega, \mathcal{F}_{T},\left(\mathcal{F}_{t}\right)_{t \in[0, T]}\right)$, the processes $\left(Y, Z, W, W^{(1)}\right)$ in $(5)$ correspond respectively to the four components of $\omega$ and are formally functions of $\omega$. We assume that processes $Y, Z$ are adapted to the filtration $\left\{\mathcal{F}_{t}\right\}_{t \in[0, T]}$, as are $W, W^{(1)}$, which are assumed to be Brownian motions with respect to the same filtration.

\section{B Online Supplement: Proofs of the Examples in Section 5}

\section{B.1 Proof of Proposition 5.1 in the Heston model}

Proof. We first establish the following lemma.

\footnotetext{
${ }^{18}$ Continuity where the function takes the value $\infty$ is defined as usual through a compactification: if $\lim _{t \rightarrow t_{0}} \omega_{2}(t)=\infty$, then $\omega_{2}$ is continuous at $t_{0}$.
} 


\section{Lemma B.1.}

$$
\begin{gathered}
\int_{c}^{\infty} y^{-\alpha} e^{\gamma y} d y\left\{\begin{array}{l}
<\infty, \quad \text { if } \gamma<0 \text { or } \gamma=0, \alpha>1 \\
=\infty, \quad \text { if } \gamma>0 \text { or } \gamma=0, \alpha \leqslant 1
\end{array} \text { for } c>0\right. \\
\int_{x}^{\infty} y^{-\alpha} e^{\gamma y} d y \sim \frac{1}{-\gamma} x^{-\alpha} e^{\gamma x}, \quad \text { if } \gamma<0, \text { as } x \rightarrow \infty, \\
\int_{0}^{x} y^{-\alpha} e^{\gamma y} d y \begin{cases}<\infty, & \text { if } \alpha<1, \\
=\infty, & \text { if } \alpha \geqslant 1 .\end{cases} \\
\int_{0}^{x} y^{-\alpha} e^{\gamma y} d y \sim \begin{cases}\frac{x^{1-\alpha}}{\alpha-1}, & \text { if } \alpha>1, \\
\ln (x), & \text { if } \alpha=1 .\end{cases}
\end{gathered}
$$

Proof. Let us verify (36) and (37) for example. From L'Hôpital's rule, since the numerator and denominator both approach 0 if $\gamma<0$,

$$
\lim _{x \rightarrow \infty} \frac{\int_{x}^{\infty} y^{-\alpha} e^{\gamma y} d y}{x^{-\alpha} e^{\gamma x}}=\lim _{x \rightarrow \infty} \frac{-x^{-\alpha} e^{\gamma x}}{\left(\gamma x^{-\alpha}-\alpha x^{-\alpha-1}\right) e^{\gamma x}}=\lim _{x \rightarrow \infty} \frac{-1}{\gamma-\alpha / x}=-\frac{1}{\gamma} .
$$

The other asymptotics are similarly obtained.

Then we will check the conditions of Proposition 4.1. Here

$$
\widetilde{v}(x) \equiv \frac{2}{\xi^{2}} \int_{c}^{x} y^{\alpha-1} e^{-\gamma y}\left(\int_{y}^{x} z^{-\alpha} e^{\gamma z} d z\right) d y, \quad \widetilde{v}_{b}(x) \equiv \frac{2}{\xi^{2}} \int_{c}^{x} y^{\alpha} e^{-\gamma y}\left(\int_{y}^{x} z^{-\alpha} e^{\gamma z} d z\right) d y .
$$

It follows from the Lemma B.1 that $\widetilde{s}(0)$ is finite if and only if $\alpha<1$ and $\widetilde{s}(\infty)$ is finite if and only if $\gamma<0$ or $\gamma=0$ and $\alpha>1$. We consider several cases.

- $\alpha=\frac{2 \kappa \theta}{\xi^{2}}>1, \gamma>0$. In this case, $\widetilde{s}(0)=-\infty, \widetilde{v}(0)=\infty$ and $\widetilde{v}_{b}(0)=\infty, \widetilde{s}(\infty)=\infty$ so that $\widetilde{v}(\infty)=\infty$. Therefore using Proposition 4.1, (1) $E\left(S_{T}\right)=1$ since $\widetilde{v}(\infty)=\infty$ holds.

- $\alpha>1, \gamma=0$. In this case $\widetilde{s}(0)=-\infty, \widetilde{v}(0)=\infty$ and $\widetilde{v}_{b}(0)=\infty$. Moreover

$$
\widetilde{v}(\infty) \equiv \frac{2}{\xi^{2}} \int_{c}^{\infty} y^{\alpha-1}\left(\int_{y}^{\infty} z^{-\alpha} d z\right) d y=C \int_{c}^{\infty} y^{\alpha-1} y^{1-\alpha} d y=\infty
$$

Again from Proposition 4.1, (i) $E\left(S_{T}\right)=1$ if and only if either $\widetilde{v}(\infty)=\infty$ holds or $\widetilde{v}_{b}(\infty)<$ $\infty$.

- $\alpha>1, \gamma<0$. In this case $\widetilde{s}(0)=-\infty, \widetilde{v}(0)=\infty$ and $\widetilde{v}_{b}(0)=\infty$. Again we will show that $\widetilde{v}(\infty)=\infty$. By the Lemma B.1, for some positive constant $C$,

$$
\widetilde{v}(\infty) \equiv \frac{2}{\xi^{2}} \int_{c}^{\infty} y^{\alpha-1} e^{-\gamma y}\left(\int_{y}^{\infty} z^{-\alpha} e^{\gamma z} d z\right) d y \geqslant C \int_{c}^{\infty} y^{\alpha-1} e^{-\gamma y} y^{-\alpha} e^{\gamma y} d y=\infty
$$

- $\alpha=\frac{2 \kappa \theta}{\xi^{2}}=1, \gamma \geqslant 0$. In this case $\widetilde{s}(0)=-\infty$ since the integral $\int_{0}^{c} y^{-\alpha} e^{\gamma y} d y$ is divergent at 0 , so that $\widetilde{v}(0)=\infty$. Also since $\gamma \geqslant 0, \widetilde{s}(\infty)=C_{2} \int_{c}^{\infty} y^{-\alpha} e^{\gamma y} d y \geqslant C_{2} \int_{c}^{\infty} y^{-\alpha} d y=\infty$ so that $\widetilde{v}(\infty)=\infty$ and Proposition 4.1 (1) applies. 
- $\alpha=\frac{2 \kappa \theta}{\xi^{2}}=1, \gamma<0$. Here $\widetilde{s}(0)=-\infty$ since the integral $\int_{0}^{c} y^{-1} e^{\gamma y} d y$ diverges around 0 so that $\widetilde{v}(0)=\infty$. However $\widetilde{s}(\infty)=C \int_{c}^{\infty} y^{-1} e^{\gamma y} d y<\infty$. Then

$$
\widetilde{v}(\infty) \equiv \frac{2}{\xi^{2}} \int_{c}^{\infty} e^{-\gamma y}\left(\int_{y}^{\infty} z^{-1} e^{\gamma z} d z\right) d y \geqslant C \int_{c}^{\infty} e^{-\gamma y} y^{-1} e^{\gamma y} d y=\infty
$$

so again in this case $\widetilde{v}(0)=\widetilde{v}(\infty)=\infty$ and Proposition 4.1 (1) applies.

- $\alpha=\frac{2 \kappa \theta}{\xi^{2}}<1, \gamma \geqslant 0$. In this case $\widetilde{s}(0)$ is finite since the integral $\int_{0}^{c} y^{-\alpha} e^{\gamma y} d y$ is convergent at 0 . Also since $\gamma \geqslant 0, \widetilde{s}(\infty)=C_{2} \int_{c}^{\infty} y^{-\alpha} e^{\gamma y} d y \geqslant C_{2} \int_{c}^{\infty} y^{-\alpha} d y=\infty$ so that $\widetilde{v}(\infty)=\infty$. In this case, from the Lemma B.1, $\int_{0}^{y} z^{-\alpha} e^{\gamma z} d z \sim \frac{y^{1-\alpha}}{1-\alpha}$ and

$$
\widetilde{v}(0) \equiv \frac{2}{\xi^{2}} \int_{0}^{c} y^{\alpha-1} e^{-\gamma y}\left(\int_{0}^{y} z^{-\alpha} e^{\gamma z} d z\right) d y \leqslant C \int_{0}^{c} y^{\alpha-1} e^{-\gamma y} \frac{y^{1-\alpha}}{1-\alpha} d y<\infty
$$

Similarly,

$$
\widetilde{v}_{b}(0) \equiv \frac{2}{\xi^{2}} \int_{0}^{c} y^{\alpha} e^{-\gamma y}\left(\int_{0}^{y} z^{-\alpha} e^{\gamma z} d z\right) d y \leqslant C \int_{0}^{c} y^{\alpha} e^{-\gamma y} \frac{y^{1-\alpha}}{1-\alpha} d y<\infty
$$

So in this case $\widetilde{v}(\infty)=\infty$ and $\widetilde{v}_{b}(0)<\infty$ so that Proposition 4.1 (3) applies.

- $\alpha=\frac{2 \kappa \theta}{\xi^{2}}<1, \gamma<0$. In this case $\widetilde{s}(0)$ is finite since the integral $\int_{0}^{c} y^{-\alpha} e^{\gamma y} d y$ is convergent at 0 . Also

$$
\begin{aligned}
\widetilde{v}(0) & \equiv \frac{2}{\xi^{2}} \int_{0}^{c} y^{\alpha-1} e^{-\gamma y}\left(\int_{0}^{y} z^{-\alpha} e^{\gamma z} d z\right) d y \leqslant C \int_{0}^{c} y^{\alpha-1} e^{-\gamma y} \frac{y^{1-\alpha}}{1-\alpha} d y<\infty \\
\widetilde{v}_{b}(0) & \equiv \frac{2}{\xi^{2}} \int_{0}^{c} y^{\alpha} e^{-\gamma y}\left(\int_{0}^{y} z^{-\alpha} e^{\gamma z} d z\right) d y \leqslant C \int_{0}^{c} y^{\alpha} e^{-\gamma y} \frac{y^{1-\alpha}}{1-\alpha} d y<\infty
\end{aligned}
$$

Also, since $\gamma<0, \widetilde{s}(\infty)=C_{2} \int_{c}^{\infty} y^{-\alpha} e^{\gamma y} d y<\infty$. Then

$$
\widetilde{v}(\infty) \equiv \frac{2}{\xi^{2}} \int_{c}^{\infty} y^{\alpha-1} e^{-\gamma y}\left(\int_{y}^{\infty} z^{-\alpha} e^{\gamma z} d z\right) d y \geqslant C \int_{c}^{\infty} y^{\alpha-1} e^{-\gamma y} y^{-\alpha} e^{\gamma y} d y=\infty
$$

so that $\widetilde{v}(\infty)=\infty$. In this case $\widetilde{v}_{b}(0)<\infty$ and $\widetilde{v}(\infty)=\infty$ so that Proposition 4.1 (3) applies.

In summary, for the Heston model, $\left\{S_{t}\right\}_{t \leqslant T}$ is always a martingale.

\section{B.2 Proof of Proposition 5.2 in the Heston model}

Proof. We will check the conditions of Proposition 4.2.

It follows from the Lemma B.1 that $\widetilde{s}(0)$ is finite if and only if $\alpha<1$ and $\widetilde{s}(\infty)$ is finite if and only if $\gamma<0$ or $\gamma=0$ and $\alpha>1$. Note that

$$
\widetilde{v}_{b}(\infty) \equiv \frac{2}{\xi^{2}} \int_{c}^{\infty} y^{\alpha} e^{-\gamma y}\left(\int_{y}^{\infty} z^{-\alpha} e^{\gamma z} d z\right) d y
$$


Also,

$$
\widetilde{v}_{b}(0) \equiv \frac{2}{\xi^{2}} \int_{0}^{c} y^{\alpha} e^{-\gamma y}\left(\int_{0}^{y} z^{-\alpha} e^{\gamma z} d z\right) d y<\infty
$$

if and only if $\alpha<1$.

Again consider several cases.

- $\alpha>1, \gamma>0$. In this case $\widetilde{s}(0)=-\infty$ and $\widetilde{v}_{b}(0)=\infty, \widetilde{s}(\infty)=\infty$ so that $\widetilde{v}_{b}(\infty)=\infty$. Therefore, since $\widetilde{v}_{b}(0)=\widetilde{v}_{b}(\infty)=\infty$, none of the conditions of Proposition 4.2 apply.

- $\alpha>1, \gamma=0$. In this case, $\widetilde{s}(0)=-\infty, \widetilde{v}_{b}(0)=\infty$. Moreover,

$$
\widetilde{v}_{b}(\infty) \equiv \frac{2}{\xi^{2}} \int_{c}^{\infty} y^{\alpha}\left(\int_{y}^{\infty} z^{-\alpha} d z\right) d y=C \int_{c}^{\infty} y^{\alpha} y^{1-\alpha} d y=\infty .
$$

Again, since $\widetilde{v}_{b}(0)=\widetilde{v}_{b}(\infty)=\infty$, none of the conditions of Proposition 4.2 apply.

- $\alpha>1, \gamma<0$. In this case $\widetilde{s}(0)=-\infty$, and $\widetilde{v}_{b}(0)=\infty$. Again we will show that $\widetilde{v}_{b}(\infty)=\infty$. By the Lemma B.1, for some positive constant $C$,

$$
\widetilde{v}_{b}(\infty) \equiv \frac{2}{\xi^{2}} \int_{c}^{\infty} y^{\alpha} e^{-\gamma y}\left(\int_{y}^{\infty} z^{-\alpha} e^{\gamma z} d z\right) d y \geqslant C \int_{c}^{\infty} y^{\alpha} e^{-\gamma y} y^{-\alpha} e^{\gamma y} d y=C \int_{c}^{\infty} 1 d y=\infty
$$

Again, since $\widetilde{v}_{b}(0)=\widetilde{v}_{b}(\infty)=\infty$, none of the conditions of Proposition 4.2 apply.

- $\alpha=1, \gamma \geqslant 0$. In this case $\widetilde{s}(0)=-\infty$ since the integral $\int_{0}^{c} y^{-\alpha} e^{\gamma y} d y$ is divergent at 0 , so that $\widetilde{v}_{b}(0)=\infty$. Also since $\gamma \geqslant 0, \widetilde{s}(\infty)=C_{2} \int_{c}^{\infty} y^{-\alpha} e^{\gamma y} d y \geqslant C_{2} \int_{c}^{\infty} y^{-\alpha} d y=\infty$ so that $\widetilde{v}_{b}(\infty)=\infty$. Since $\widetilde{v}_{b}(0)=\widetilde{v}_{b}(\infty)=\infty$, none of the conditions of Proposition 4.2 apply.

- $\alpha=1, \gamma<0$. Here $\widetilde{s}(0)=-\infty$ since the integral $\int_{0}^{c} y^{-1} e^{\gamma y} d y$ diverges around 0 so that $\widetilde{v}_{b}(0)=\infty$. However $\widetilde{s}(\infty)=C \int_{c}^{\infty} y^{-1} e^{\gamma y} d y<\infty$. Then

$$
\widetilde{v}_{b}(\infty) \equiv \frac{2}{\xi^{2}} \int_{c}^{\infty} y^{1} e^{-\gamma y}\left(\int_{y}^{\infty} z^{-1} e^{\gamma z} d z\right) d y \geqslant C \int_{c}^{\infty} y e^{-\gamma y} y^{-1} e^{\gamma y} d y=\infty .
$$

Since $\widetilde{v}_{b}(0)=\widetilde{v}_{b}(\infty)=\infty$, none of the conditions of Proposition 4.2 apply.

- $\alpha<1, \gamma \geqslant 0$. In this case $\widetilde{s}(0)$ is finite since the integral $\int_{0}^{c} y^{-\alpha} e^{\gamma y} d y$ is convergent at 0 . Also since $\gamma \geqslant 0, \widetilde{s}(\infty)=C_{2} \int_{c}^{\infty} y^{-\alpha} e^{\gamma y} d y \geqslant C_{2} \int_{c}^{\infty} y^{-\alpha} d y=\infty$ so that $\widetilde{v}_{b}(\infty)=\infty$. In this case, from the Lemma B.1, $\int_{0}^{y} z^{-\alpha} e^{\gamma z} d z \sim \frac{y^{1-\alpha}}{1-\alpha}$ and

$$
\widetilde{v}_{b}(0) \equiv \frac{2}{\xi^{2}} \int_{0}^{c} y^{\alpha} e^{-\gamma y}\left(\int_{0}^{y} z^{-\alpha} e^{\gamma z} d z\right) d y \leqslant C \int_{0}^{c} y^{\alpha} e^{-\gamma y} \frac{y^{1-\alpha}}{1-\alpha} d y<\infty
$$

In this case $\widetilde{v}_{b}(0)<\infty$ and $\widetilde{s}(\infty)=\infty$ so that the condition $C^{\prime}$ of Proposition 4.2 is satisfied.

- $\alpha<1, \gamma<0$. In this case $\widetilde{s}(0)$ is finite since the integral $\int_{0}^{c} y^{-\alpha} e^{\gamma y} d y$ is convergent at 0 . Also

$$
\widetilde{v}_{b}(0) \equiv \frac{2}{\xi^{2}} \int_{0}^{c} y^{\alpha} e^{-\gamma y}\left(\int_{0}^{y} z^{-\alpha} e^{\gamma z} d z\right) d y \leqslant C \int_{0}^{c} y^{\alpha} e^{-\gamma y} \frac{y^{1-\alpha}}{1-\alpha} d y<\infty
$$


and $\widetilde{s}(\infty)=C_{2} \int_{c}^{\infty} y^{-\alpha} e^{\gamma y} d y<\infty$. Therefore, condition $D^{\prime}$ of Proposition 4.2 holds if and only if $\widetilde{v}_{b}(\infty)<\infty$. But

$$
\widetilde{v}_{b}(\infty) \equiv \frac{2}{\xi^{2}} \int_{c}^{\infty} y^{\alpha} e^{-\gamma y}\left(\int_{y}^{\infty} z^{-\alpha} e^{\gamma z} d z\right) d y \geqslant C \int_{c}^{\infty} y^{\alpha} e^{-\gamma y} y^{-\alpha} e^{\gamma y} d y=C \int_{c}^{\infty} 1 d y=\infty
$$

so the conditions of Proposition 4.2 fail.

In summary, for the Heston model, $\left\{S_{t} ; t \leqslant \infty\right\}$ is a uniformly integrable martingale if and only if $\alpha=\frac{2 \kappa \theta}{\xi^{2}}<1$, and $\gamma=2 \frac{\kappa-\rho \xi}{\xi^{2}} \geqslant 0$, i.e. if and only if $\rho \xi \leqslant \kappa<\frac{\xi^{2}}{2 \theta}$.

\section{B.3 Proof of Lemma 5.1}

Proof. For the right boundary $r$, divide into two cases:

(i) When $a<-1, \lim _{y \rightarrow \infty} y^{a+1} \exp \left(\frac{d}{y}\right)=0$. From L'Hôpital's rule

$$
\lim _{y \rightarrow \infty} \frac{\int_{y}^{\infty} z^{a} \exp \left(\frac{d}{z}\right) d z}{y^{a+1} \exp \left(\frac{d}{y}\right)}=-\frac{1}{a+1}>0 .
$$

Since $\int_{y}^{\infty} z^{a} \exp \left(\frac{d}{z}\right) d z$ is decreasing in $y$, there exists $M>c>0$, such that for $y>M$

$$
\int_{y}^{\infty} z^{a} \exp \left(\frac{d}{z}\right) d z<\frac{-2}{a+1} y^{a+1} \exp \left(\frac{d}{y}\right) .
$$

Substitute (43) into (28) with $x=\infty$

$$
\begin{aligned}
v(\infty) & =\frac{2}{\xi^{2}} \int_{c}^{\infty} \frac{1}{y^{a+3} \exp \left(\frac{d}{y}\right)}\left(\int_{y}^{\infty} z^{a} \exp \left(\frac{d}{z}\right) d z\right) d y \\
& =\frac{2}{\xi^{2}} \int_{c}^{M} \frac{\int_{y}^{\infty} z^{a} \exp \left(\frac{d}{z}\right) d z}{y^{a+3} \exp \left(\frac{d}{y}\right)} d y+\frac{2}{\xi^{2}} \int_{M}^{\infty} \frac{\int_{y}^{\infty} z^{a} \exp \left(\frac{d}{z}\right) d z}{y^{a+3} \exp \left(\frac{d}{y}\right)} d y \\
& <\frac{2}{\xi^{2}} \int_{c}^{M} \frac{\int_{y}^{\infty} z^{a} \exp \left(\frac{d}{z}\right) d z}{y^{a+3} \exp \left(\frac{d}{y}\right)} d y+\frac{2}{\xi^{2}} \int_{M}^{\infty} \frac{1}{y^{a+3} \exp \left(\frac{d}{y}\right)} \frac{-2}{a+1} y^{a+1} \exp \left(\frac{d}{y}\right) d y \\
& =\frac{2}{\xi^{2}} \int_{c}^{M} \frac{1}{y^{a+3} \exp \left(\frac{d}{y}\right)}\left(\int_{y}^{\infty} z^{a} \exp \left(\frac{d}{z}\right) d z\right) d y+\frac{-4}{(a+1) \xi^{2}} \int_{M}^{\infty} \frac{1}{y^{2}} d y \\
& =\frac{2}{\xi^{2}} \int_{c}^{M} \frac{1}{y^{a+3} \exp \left(\frac{d}{y}\right)} \int_{y}^{\infty} z^{a} \exp \left(\frac{d}{z}\right) d z d y+\frac{-4}{(a+1) \xi^{2} M} \\
& <\infty .
\end{aligned}
$$

From (42), there exists $M^{\prime}>c>0$, such that for $y>M^{\prime}$

$$
\int_{y}^{\infty} z^{a} \exp \left(\frac{d}{z}\right) d z>\frac{-1}{2(a+1)} y^{a+1} \exp \left(\frac{d}{y}\right) .
$$


Similarly substitute (44) into (29) with $x=\infty$

$$
\begin{aligned}
v_{b}(\infty) & =\frac{2}{\xi^{2}} \int_{c}^{\infty} \frac{1}{y^{a+2} \exp \left(\frac{d}{y}\right)}\left(\int_{y}^{\infty} z^{a} \exp \left(\frac{d}{z}\right) d z\right) d y \\
& \geqslant \frac{2}{\xi^{2}} \int_{M^{\prime}}^{\infty} \frac{1}{y^{a+2} \exp \left(\frac{d}{y}\right)}\left(\int_{y}^{\infty} z^{a} \exp \left(\frac{d}{z}\right) d z\right) d y \\
& >\frac{2}{\xi^{2}} \int_{M^{\prime}}^{\infty} \frac{1}{y^{a+2} \exp \left(\frac{d}{y}\right)}\left(\frac{-1}{2(a+1)} y^{a+1} \exp \left(\frac{d}{y}\right)\right) d y \\
& =\frac{-1}{\xi^{2}(a+1)} \int_{M^{\prime}}^{\infty} \frac{1}{y} d y \\
& =\infty
\end{aligned}
$$

(ii) When $a \geqslant-1$, since $d>0$, we have that $\exp \left(\frac{d}{y}\right) \geqslant 1$, for $y>c>0$. Then

$$
s(\infty)=\frac{b}{c^{a}} \int_{c}^{\infty} y^{a} \exp \left(\frac{d}{y}\right) d y \geqslant \frac{b}{c^{a}} \int_{c}^{\infty} y^{a} d y=\infty .
$$

Thus $v(\infty)=\infty$ and $v_{b}(\infty)=\infty$ in this case. To summarize, $v(r)<\infty$ if and only if $a<-1$, and $v_{b}(r)=\infty$ for $a \in \mathbb{R}$.

For the left endpoint $\ell$

$$
v(0)=\frac{2}{\xi^{2}} \int_{0}^{c} \frac{1}{y^{a+3} \exp \left(\frac{d}{y}\right)}\left(\int_{0}^{y} z^{a} \exp \left(\frac{d}{z}\right) d z\right) d y
$$

and

$$
v_{b}(0)=\frac{2}{\xi^{2}} \int_{0}^{c} \frac{1}{y^{a+2} \exp \left(\frac{d}{y}\right)}\left(\int_{0}^{y} z^{a} \exp \left(\frac{d}{z}\right) d z\right) d y
$$

For $0 \leqslant z \leqslant y$, we have $e^{\frac{d}{z}} \geqslant e^{\frac{d}{y}}$, and substitute this inequality into (45)

$$
v(0) \geqslant \frac{2}{\xi^{2}} \int_{0}^{c} \frac{1}{y^{a+3} \exp \left(\frac{d}{y}\right)}\left(\int_{0}^{y} z^{a} d z\right) \exp \left(\frac{d}{y}\right) d y=\frac{2}{(a+1) \xi^{2}} \int_{0}^{c} \frac{1}{y^{2}} d y=\infty .
$$

Similarly substitute this inequality into (46)

$$
\begin{aligned}
v_{b}(0) & \geqslant \frac{2}{\xi^{2}} \int_{0}^{c} \frac{1}{y^{a+2} \exp \left(\frac{d}{y}\right)}\left(\int_{0}^{y} z^{a} d z\right) \exp \left(\frac{d}{y}\right) d y \\
& =\frac{2}{(a+1) \xi^{2}} \int_{0}^{c} \frac{1}{y} d y=\infty
\end{aligned}
$$

To summarize, $v(\ell)=\infty$ and $v_{b}(\ell)=\infty$ for $a \in \mathbb{R}$. From $(27)$, the above proofs also work for the case of $\widetilde{v}$ by substituting $a$ for $\widetilde{a}$. 


\section{B.4 Proof of Proposition 5.7}

Proof. Divide into three cases:

(i) When $\alpha>0, \widetilde{s}(\infty)=\infty$, then $\widetilde{v}(\infty)=\infty$ and $\widetilde{v}_{b}(\infty)=\infty$.

(ii) When $\alpha=0$

$$
\widetilde{v}(x)=\frac{1}{\kappa \theta} \int_{c}^{x}\left(1-e^{-\frac{2 \kappa \theta}{\gamma^{2}}(x-y)}\right) d y=\frac{1}{\kappa \theta}\left(x+\frac{\gamma^{2}}{2 \kappa \theta} e^{\frac{2 \kappa \theta}{\gamma^{2}}(c-x)}-c-\frac{\gamma^{2}}{2 \kappa \theta}\right) .
$$

Then $\widetilde{v}(\infty)=\infty$. Similarly we can compute

$$
\widetilde{v}_{b}(x)=\frac{1}{\kappa \theta} \int_{c}^{x} y^{2}\left(1-e^{-\frac{2 \kappa \theta}{\gamma^{2}}(x-y)}\right) d y=\frac{1}{3 \kappa \theta} x^{3}-e^{-\frac{2 \kappa \theta}{\gamma^{2}} x} \int_{c}^{x} y^{2} e^{\frac{2 \kappa \theta}{\gamma^{2}} y} d y-\frac{c^{3}}{3 \kappa \theta} .
$$

Since $\int_{c}^{x} y^{2} e^{\frac{2 \kappa \theta}{\gamma^{2}} y} d y \leqslant \int_{c}^{x} x^{2} e^{\frac{2 \kappa \theta}{\gamma^{2}} y} d y$, then

$$
\widetilde{v}_{b}(x) \geqslant \frac{1}{3 \kappa \theta} x^{3}-e^{-\frac{2 \kappa \theta}{\gamma^{2}} x} \int_{c}^{x} x^{2} e^{\frac{2 \kappa \theta}{\gamma^{2}} y} d y-\frac{c^{3}}{3 \kappa \theta}=\frac{1}{3 \kappa \theta} x^{3}-\frac{\gamma^{2}}{2 \kappa \theta} x^{2}\left(1-e^{\frac{2 \kappa \theta}{\gamma^{2}}(c-x)}\right)-\frac{c^{3}}{3 \kappa \theta} .
$$

Then $\widetilde{v}_{b}(\infty)=\infty$ can be verified, because the right hand side of (47) tends to $\infty$ as $x \rightarrow \infty$.

(iii) When $\alpha<0$, the test function is

$$
\widetilde{v}(x)=\frac{2}{\gamma^{2}} \int_{c}^{x} \frac{\int_{y}^{x} \frac{\alpha}{\gamma^{2}}\left(z-\frac{\kappa \theta}{\alpha}\right)^{2} d z}{e^{\frac{\alpha}{\gamma^{2}}\left(y-\frac{\kappa \theta}{\alpha}\right)^{2}}} d y=\frac{2}{\gamma^{2}} \int_{c}^{x} e^{-\frac{\alpha}{\gamma^{2}}\left(y-\frac{\kappa \theta}{\alpha}\right)^{2}}\left(\int_{y}^{x} \frac{\alpha}{\gamma^{2}}\left(z-\frac{\kappa \theta}{\alpha}\right)^{2} d z\right) d y .
$$

Then

$$
\widetilde{v}(\infty)=\frac{2}{\gamma^{2}} \int_{c}^{\infty} e^{-\frac{\alpha}{\gamma^{2}}\left(y-\frac{\kappa \theta}{\alpha}\right)^{2}}\left(\int_{y}^{\infty} \frac{\alpha}{\gamma^{2}}\left(z-\frac{\kappa \theta}{\alpha}\right)^{2} d z\right) d y
$$

Since $\alpha<0$ is assumed here, then $\lim _{y \rightarrow \infty} y^{-1} e^{\frac{\alpha}{\gamma^{2}}\left(y-\frac{\kappa \theta}{\alpha}\right)^{2}}=0$, and we can apply L'Hôpital's rule

$$
\lim _{y \rightarrow \infty} \frac{\int_{y}^{\infty} \frac{\alpha}{\gamma^{2}}\left(z-\frac{\kappa \theta}{\alpha}\right)^{2} d z}{y^{-1} e^{\frac{\alpha}{\gamma^{2}}\left(y-\frac{\kappa \theta}{\alpha}\right)^{2}}}=\frac{-\gamma^{2}}{2 \alpha}>0 .
$$

So as $y \rightarrow \infty$, there exists $M>c>0$, such that for $y>M$

$$
\int_{y}^{\infty} \frac{\alpha}{\gamma^{2}}\left(z-\frac{\kappa \theta}{\alpha}\right)^{2} d z>\frac{-\gamma^{2}}{4 \alpha} y^{-1} e^{\frac{\alpha}{\gamma^{2}}\left(y-\frac{\kappa \theta}{\alpha}\right)^{2}} .
$$

Substitute (49) into (48)

$$
\begin{aligned}
\widetilde{v}(\infty) & \geqslant \frac{2}{\gamma^{2}} \int_{M}^{\infty} e^{-\frac{\alpha}{\gamma^{2}}\left(y-\frac{\kappa \theta}{\alpha}\right)^{2}}\left(\int_{y}^{\infty} \frac{\alpha}{\gamma^{2}}\left(z-\frac{\kappa \theta}{\alpha}\right)^{2} d z\right) d y \\
& >\frac{2}{\gamma^{2}} \int_{M}^{\infty} e^{-\frac{\alpha}{\gamma^{2}}\left(y-\frac{\kappa \theta}{\alpha}\right)^{2}}\left(\frac{-\gamma^{2}}{4 \alpha} y^{-1} e^{\frac{\alpha}{\gamma^{2}}\left(y-\frac{\kappa \theta}{\alpha}\right)^{2}}\right) d y \\
& =\frac{-1}{2 \alpha} \int_{M}^{\infty} y^{-1} d y=\infty .
\end{aligned}
$$


Thus $\widetilde{v}(\infty)=\infty$ in this case. Similarly, we can compute

$$
\widetilde{v}_{b}(\infty)=\frac{2}{\gamma^{2}} \int_{c}^{\infty} y^{2} e^{-\frac{\alpha}{\gamma^{2}}\left(y-\frac{\kappa \theta}{\alpha}\right)^{2}}\left(\int_{y}^{\infty} \frac{\alpha}{\gamma^{2}}\left(z-\frac{\kappa \theta}{\alpha}\right)^{2} d z\right) d y
$$

With the same $M$ as above, substitute (49) into (50)

$$
\begin{aligned}
\widetilde{v}_{b}(\infty) & \geqslant \frac{2}{\gamma^{2}} \int_{M}^{\infty} y^{2} e^{-\frac{\alpha}{\gamma^{2}}\left(y-\frac{\kappa \theta}{\alpha}\right)^{2}}\left(\int_{y}^{\infty} \frac{\alpha}{\gamma^{2}}\left(z-\frac{\kappa \theta}{\alpha}\right)^{2} d z\right) d y \\
& >\frac{2}{\gamma^{2}} \int_{c}^{\infty} y^{2} e^{-\frac{\alpha}{\gamma^{2}}\left(y-\frac{\kappa \theta}{\alpha}\right)^{2}}\left(\frac{-\gamma^{2}}{4 \alpha} y^{-1} e^{\frac{\alpha}{\gamma^{2}}\left(y-\frac{\kappa \theta}{\alpha}\right)^{2}}\right) d y \\
& =\frac{-1}{2 \alpha} \int_{M}^{\infty} y d y=\infty
\end{aligned}
$$

Thus $\widetilde{v}_{b}(\infty)=\infty$ in this case.

We then consider the case of the left endpoint $\ell$. From the definition of $\widetilde{s}(\cdot)$, we have that $\widetilde{s}(0)>-\infty$ for $\alpha \in \mathbb{R}$.

Similarly as above, we consider the following two cases:

(i) When $\alpha=0, \widetilde{v}(0)=\frac{1}{\kappa \theta}\left(\frac{\gamma^{2}}{2 \kappa \theta} e^{\frac{2 \kappa \theta}{\gamma^{2}}(c)}-c-\frac{\gamma^{2}}{2 \kappa \theta}\right)<\infty$.

(ii) When $\alpha \neq 0$,

$$
\widetilde{v}(0)=\frac{2}{\gamma^{2}} \int_{0}^{c} e^{-\frac{\alpha}{\gamma^{2}}\left(y-\frac{\kappa \theta}{\alpha}\right)^{2}}\left(\int_{0}^{y} \frac{\alpha}{\gamma^{2}}\left(z-\frac{\kappa \theta}{\alpha}\right)^{2} d z\right) d y .
$$

Since $\lim _{y \rightarrow 0} y e^{\frac{\alpha}{\gamma^{2}}\left(y-\frac{\kappa \theta}{\alpha}\right)^{2}}=0$, we can apply L'Hôpital's rule

$$
\lim _{y \rightarrow 0} \frac{\int_{0}^{y} \frac{\alpha}{\gamma^{2}}\left(z-\frac{\kappa \theta}{\alpha}\right)^{2} d z}{y e^{\frac{\alpha}{\gamma^{2}}\left(y-\frac{\kappa \theta}{\alpha}\right)^{2}}}=1 .
$$

So as $y \rightarrow 0$, there exists $0<\varepsilon<c$, such that for $0 \leqslant y<\varepsilon$

$$
\int_{0}^{y} \frac{\alpha}{\gamma^{2}}\left(z-\frac{\kappa \theta}{\alpha}\right)^{2} d z<2 y e^{\frac{\alpha}{\gamma^{2}}\left(y-\frac{\kappa \theta}{\alpha}\right)^{2}} .
$$

Substitute (52) into (51)

$$
\begin{aligned}
\widetilde{v}(0)= & \frac{2}{\gamma^{2}} \int_{0}^{\varepsilon} e^{-\frac{\alpha}{\gamma^{2}}\left(y-\frac{\kappa \theta}{\alpha}\right)^{2}}\left(\int_{0}^{y} \frac{\alpha}{\gamma^{2}}\left(z-\frac{\kappa \theta}{\alpha}\right)^{2} d z\right) d y \\
& +\frac{2}{\gamma^{2}} \int_{\varepsilon}^{c} e^{-\frac{\alpha}{\gamma^{2}}\left(y-\frac{\kappa \theta}{\alpha}\right)^{2}}\left(\int_{0}^{y} \frac{\alpha}{\gamma^{2}}\left(z-\frac{\kappa \theta}{\alpha}\right)^{2} d z\right) d y \\
< & \frac{2}{\gamma^{2}} \int_{0}^{\varepsilon} e^{-\frac{\alpha}{\gamma^{2}}\left(y-\frac{\kappa \theta}{\alpha}\right)^{2}}\left(2 y e^{\frac{\alpha}{\gamma^{2}}\left(y-\frac{\kappa \theta}{\alpha}\right)^{2}}\right) d y \\
& +\frac{2}{\gamma^{2}} \int_{\varepsilon}^{c} e^{-\frac{\alpha}{\gamma^{2}}\left(y-\frac{\kappa \theta}{\alpha}\right)^{2}}\left(\int_{0}^{y} \frac{\alpha}{\gamma^{2}}\left(z-\frac{\kappa \theta}{\alpha}\right)^{2} d z\right) d y
\end{aligned}
$$


Then,

$$
\begin{aligned}
\widetilde{v}(\infty) & <\frac{2}{\gamma^{2}} \int_{0}^{\varepsilon} 2 y d y+\frac{2}{\gamma^{2}} \int_{\varepsilon}^{c} e^{-\frac{\alpha}{\gamma^{2}}\left(y-\frac{\kappa \theta}{\alpha}\right)^{2}}\left(\int_{0}^{y} \frac{\alpha}{\gamma^{2}}\left(z-\frac{\kappa \theta}{\alpha}\right)^{2} d z\right) d y \\
& =\frac{2 \varepsilon^{2}}{\gamma^{2}}+\frac{2}{\gamma^{2}} \int_{\varepsilon}^{c} e^{-\frac{\alpha}{\gamma^{2}}\left(y-\frac{\kappa \theta}{\alpha}\right)^{2}}\left(\int_{0}^{y} \frac{\alpha}{\gamma^{2}}\left(z-\frac{\kappa \theta}{\alpha}\right)^{2} d z\right) d y \\
& <\infty
\end{aligned}
$$

To summarize, $\widetilde{v}(\ell)<\infty$ for $\alpha \in \mathbb{R}$. Similarly, when $\alpha=0, \widetilde{v}_{b}(0)=\int_{0}^{c} y^{2} e^{\frac{2 \kappa \theta}{\gamma^{2}} y} d y-\frac{c^{3}}{3 \kappa \theta}<\infty$.

When $\alpha \neq 0$,

$$
\widetilde{v}_{b}(0)=\frac{2}{\gamma^{2}} \int_{0}^{c} y^{2} e^{-\frac{\alpha}{\gamma^{2}}\left(y-\frac{\kappa \theta}{\alpha}\right)^{2}}\left(\int_{0}^{y} \frac{\alpha}{\gamma^{2}}\left(z-\frac{\kappa \theta}{\alpha}\right)^{2} d z\right) d y .
$$

Substitute (52) into (54), and use the same $\varepsilon$ as above. For $0 \leqslant y<\varepsilon$

$$
\begin{aligned}
\widetilde{v}_{b}(0)= & \frac{2}{\gamma^{2}} \int_{0}^{\varepsilon} y^{2} e^{-\frac{\alpha}{\gamma^{2}}\left(y-\frac{\kappa \theta}{\alpha}\right)^{2}}\left(\int_{0}^{y} \frac{\alpha}{\gamma^{2}}\left(z-\frac{\kappa \theta}{\alpha}\right)^{2} d z\right) d y \\
& +\frac{2}{\gamma^{2}} \int_{\varepsilon}^{c} y^{2} e^{-\frac{\alpha}{\gamma^{2}}\left(y-\frac{\kappa \theta}{\alpha}\right)^{2}}\left(\int_{0}^{y} \frac{\alpha}{\gamma^{2}}\left(z-\frac{\kappa \theta}{\alpha}\right)^{2} d z\right) d y \\
< & \frac{2}{\gamma^{2}} \int_{0}^{\varepsilon} y^{2} e^{-\frac{\alpha}{\gamma^{2}}\left(y-\frac{\kappa \theta}{\alpha}\right)^{2}}\left(2 y e^{\frac{\alpha}{\gamma^{2}}\left(y-\frac{\kappa \theta}{\alpha}\right)^{2}}\right) d y \\
& +\frac{2}{\gamma^{2}} \int_{\varepsilon}^{c} y^{2} e^{-\frac{\alpha}{\gamma^{2}}\left(y-\frac{\kappa \theta}{\alpha}\right)^{2}}\left(\int_{0}^{y} \frac{\alpha}{\gamma^{2}}\left(z-\frac{\kappa \theta}{\alpha}\right)^{2} d z\right) d y \\
= & \frac{2}{\gamma^{2}} \int_{0}^{\varepsilon} 2 y^{3} d y+\frac{2}{\gamma^{2}} \int_{\varepsilon}^{c} y^{2} e^{-\frac{\alpha}{\gamma^{2}}\left(y-\frac{\kappa \theta}{\alpha}\right)^{2}}\left(\int_{0}^{y} \frac{\alpha}{\gamma^{2}}\left(z-\frac{\kappa \theta}{\alpha}\right)^{2} d z\right) d y \\
= & \frac{\varepsilon^{4}}{\gamma^{2}}+\frac{2}{\gamma^{2}} \int_{\varepsilon}^{c} y^{2} e^{-\frac{\alpha}{\gamma^{2}}\left(y-\frac{\kappa \theta}{\alpha}\right)^{2}}\left(\int_{0}^{y} \frac{\alpha}{\gamma^{2}}\left(z-\frac{\kappa \theta}{\alpha}\right)^{2} d z\right) d y \\
< & \infty
\end{aligned}
$$

To summarize, $\widetilde{v}_{b}(\ell)<\infty$, for $\alpha \in \mathbb{R}$.

\section{B.5 Proof of Proposition 5.10}

Proof. We distinguish three situations:

(I) $\mu>\frac{1}{2} \sigma^{2}$. Apply a change of variable $z=\sqrt{y}$. Then $y=z^{2}, d y=2 z d z$, and

$$
\widetilde{s}(x)=2 C_{1} \int_{\sqrt{c}}^{\sqrt{x}} z^{1-\frac{4 \mu}{\sigma^{2}}} e^{-\frac{2 \rho}{\sigma} z} d z=2 C_{1} \int_{\sqrt{c}}^{\sqrt{x}} z^{-\alpha} e^{-\gamma z} d z, \quad x \in \bar{J} .
$$

Note that the function in (56) is similar to the scale function in (25), except that there is a $\sqrt{x}$ in place of $x$. From $(56)$

$$
\widetilde{s}(\infty)=2 C_{1} \int_{\sqrt{c}}^{\infty} z^{-\alpha} e^{-\gamma z} d z
$$


From the property of the gamma function

$$
\widetilde{s}(\infty) \begin{cases}<\infty, & \text { if } \gamma \geqslant 0, \\ =\infty, & \text { if } \gamma<0\end{cases}
$$

Divide into three cases based on $\gamma$ :

(i) When $\gamma<0, \widetilde{s}(\infty)=\infty$, then $\widetilde{v}(\infty)=\infty$ and $\widetilde{v}_{b}(\infty)=\infty$.

(ii) When $\gamma=0, \widetilde{v}(\infty)$ and $\widetilde{v}_{b}(\infty)$ can be simplified and

$$
\widetilde{v}(\infty)=\frac{2}{\sigma^{2}} \int_{c}^{\infty} y^{\frac{\alpha-3}{2}}\left(\int_{y}^{\infty} z^{-\frac{\alpha+1}{2}} d z\right) d y=\frac{4}{\sigma^{2}(\alpha-1)} \int_{c}^{\infty} y^{-1} d y=\infty
$$

and

$$
\widetilde{v}_{b}(\infty)=\frac{2}{\sigma^{2}} \int_{c}^{\infty} y^{\frac{\alpha-1}{2}}\left(\int_{y}^{\infty} z^{-\frac{\alpha+1}{2}} d z\right) d y=\int_{c}^{\infty} \frac{4}{\sigma^{2}(\alpha-1)} d y=\infty .
$$

(iii) When $\gamma>0$, from (34)

$$
\widetilde{v}(\infty)=\frac{2}{\sigma^{2}} \int_{c}^{\infty} y^{\frac{\alpha-3}{2}} e^{\gamma \sqrt{y}}\left(\int_{y}^{\infty} z^{-\frac{\alpha+1}{2}} e^{-\gamma \sqrt{z}} d z\right) d y
$$

and

$$
\widetilde{v}_{b}(\infty)=\frac{2}{\sigma^{2}} \int_{c}^{\infty} y^{\frac{\alpha-1}{2}} e^{\gamma \sqrt{y}}\left(\int_{y}^{\infty} z^{-\frac{\alpha+1}{2}} e^{-\gamma \sqrt{z}} d z\right) d y
$$

Since $\alpha>1$, then $\lim _{y \rightarrow \infty} y^{-\frac{\alpha}{2}} e^{-\gamma \sqrt{y}}=0$, and from L'Hôpital's rule

$$
\lim _{y \rightarrow \infty} \frac{\int_{y}^{\infty} z^{-\frac{\alpha+1}{2}} e^{-\gamma \sqrt{z}} d z}{y^{-\frac{\alpha}{2}} e^{-\gamma \sqrt{y}}}=\lim _{y \rightarrow \infty} \frac{1}{\frac{\alpha}{2} y^{-1 / 2}+\frac{\gamma}{2}}=\frac{2}{\gamma}>0 .
$$

As $y \rightarrow \infty$

$$
\int_{y}^{\infty} z^{-\frac{\alpha+1}{2}} e^{-\gamma \sqrt{z}} d z \sim \frac{2}{\gamma} y^{-\frac{\alpha}{2}} e^{-\gamma \sqrt{y}} .
$$

From (59), there exists $0<M<\infty$, such that for $y>M$

$$
\int_{y}^{\infty} z^{-\frac{\alpha+1}{2}} e^{-\gamma \sqrt{z}} d z<\frac{4}{\gamma} y^{-\frac{\alpha}{2}} e^{-\gamma \sqrt{y}} .
$$

Substitute (60) into (57)

$$
\begin{aligned}
\widetilde{v}(\infty) & =\frac{2}{\sigma^{2}} \int_{c}^{\infty} y^{\frac{\alpha-3}{2}} e^{\gamma \sqrt{y}}\left(\int_{y}^{\infty} z^{-\frac{\alpha+1}{2}} e^{-\gamma \sqrt{z}} d z\right) d y \\
& =\frac{2}{\sigma^{2}} \int_{c}^{M} y^{\frac{\alpha-3}{2}} e^{\gamma \sqrt{y}}\left(\int_{y}^{\infty} z^{-\frac{\alpha+1}{2}} e^{-\gamma \sqrt{z}} d z\right) d y+\frac{2}{\sigma^{2}} \int_{M}^{\infty} y^{\frac{\alpha-3}{2}} e^{\gamma \sqrt{y}}\left(\int_{y}^{\infty} z^{-\frac{\alpha+1}{2}} e^{-\gamma \sqrt{z}} d z\right) d y \\
& <\frac{2}{\sigma^{2}} \int_{c}^{M} y^{\frac{\alpha-3}{2}} e^{\gamma \sqrt{y}}\left(\int_{y}^{\infty} z^{-\frac{\alpha+1}{2}} e^{-\gamma \sqrt{z}} d z\right) d y+\frac{2}{\sigma^{2}} \int_{M}^{\infty} y^{\frac{\alpha-3}{2}} e^{\gamma \sqrt{y}}\left(\frac{4}{\gamma} y^{-\frac{\alpha}{2}} e^{-\gamma \sqrt{y}}\right) d y \\
& =\frac{2}{\sigma^{2}} \int_{c}^{M} y^{\frac{\alpha-3}{2}} e^{\gamma \sqrt{y}}\left(\int_{y}^{\infty} z^{-\frac{\alpha+1}{2}} e^{-\gamma \sqrt{z}} d z\right) d y+\frac{16}{\sqrt{M} \gamma \sigma^{2}} \\
& <\infty
\end{aligned}
$$


Then $\widetilde{v}(\infty)<\infty$, for $\gamma>0$.

From (59), there exists $0<c<M^{\prime}<\infty$, such that for $y>M^{\prime}$

$$
\int_{y}^{\infty} z^{-\frac{\alpha+1}{2}} e^{-\gamma \sqrt{z}} d z>\frac{1}{\gamma} y^{-\frac{\alpha}{2}} e^{-\gamma \sqrt{y}}
$$

Substitute (61) into (58)

$$
\begin{aligned}
\widetilde{v}(\infty) & =\frac{2}{\sigma^{2}} \int_{c}^{\infty} y^{\frac{\alpha-3}{2}} e^{\gamma \sqrt{y}}\left(\int_{y}^{\infty} z^{-\frac{\alpha+1}{2}} e^{-\gamma \sqrt{z}} d z\right) d y \\
& \geqslant \frac{2}{\sigma^{2}} \int_{M^{\prime}}^{\infty} y^{\frac{\alpha-3}{2}} e^{\gamma \sqrt{y}}\left(\int_{y}^{\infty} z^{-\frac{\alpha+1}{2}} e^{-\gamma \sqrt{z}} d z\right) d y \\
& >\frac{2}{\sigma^{2}} \int_{M^{\prime}}^{\infty} y^{\frac{\alpha-3}{2}} e^{\gamma \sqrt{y}}\left(\frac{1}{\gamma^{-\frac{\alpha}{2}}} e^{-\gamma \sqrt{y}}\right) d y \\
& =\frac{2}{\gamma \sigma^{2}} \int_{M^{\prime}}^{\infty} y^{-1} d y=\infty .
\end{aligned}
$$

Then $\widetilde{v}_{b}(\infty)=\infty$, for $\gamma>0$.

We now look at the case of the left boundary $\ell$. From (56)

$$
\widetilde{s}(0)=-2 C_{1} \int_{0}^{\sqrt{c}} z^{-\alpha} e^{-\gamma z} d z .
$$

When $\gamma>0$, since $\alpha>1$, from the property of the gamma function, we have $\widetilde{s}(0)=-\infty$. When $\gamma \leqslant 0$, then $e^{-\gamma z} \geqslant 1$, and

$$
\widetilde{s}(0)=-2 C_{1} \int_{0}^{\sqrt{c}} z^{-\alpha} e^{-\gamma z} d z \leqslant-2 C_{1} \int_{0}^{\sqrt{c}} z^{-\alpha} d z=-\infty .
$$

To summarize, $\widetilde{s}(0)=-\infty$ for $\gamma \in \mathbb{R}$. Then $\widetilde{v}(0)=\infty$ and $\widetilde{v}_{b}(0)=\infty$ hold.

(II) $\mu=\frac{1}{2} \sigma^{2}$. We consider the case when $\alpha=1$. Then

$$
\widetilde{s}(\infty)=2 C_{1} \int_{\sqrt{c}}^{\infty} z^{-1} e^{-\gamma z} d z,
$$

Divide into two cases based on the value of $\gamma$. If $\gamma \leqslant 0$, then $e^{-\gamma z} \geqslant 1$, and

$$
\widetilde{s}(\infty) \geqslant 2 C_{1} \int_{\sqrt{c}}^{\infty} z^{-1} d z=\infty
$$

Then in this case, $\widetilde{v}(r)=\infty$ and $\widetilde{v}_{b}(r)=\infty$.

If $\gamma>0$, from properties of the gamma function, $\widetilde{s}(\infty)<\infty$. To summarize, when $\alpha=1$

$$
\widetilde{s}(\infty) \begin{cases}=\infty, & \text { if } \gamma \leqslant 0, \\ <\infty, & \text { if } \gamma>0\end{cases}
$$

Similarly for the case of the left boundary $\ell$. If $\gamma>0$, from the properties of the gamma function, $\widetilde{s}(0)=-\infty$. If $\gamma \leqslant 0$, then $e^{-\gamma z} \geqslant 1$, and

$$
\widetilde{s}(0) \leqslant-2 C_{1} \int_{0}^{\sqrt{c}} z^{-1} d z=-\infty .
$$


To summarize, when $\alpha=1$, we have $\widetilde{s}(\ell)=-\infty$, then $\widetilde{v}(\ell)=\infty$ and $\widetilde{v}_{b}(\ell)=\infty$.

Consider the case when $\alpha=1$ and $\gamma>0$, from the above result, there is $\widetilde{s}(\infty)<\infty$, and we study the properties of $\widetilde{v}(\infty)$ and $\widetilde{v}_{b}(\infty)$. From the definition in $(34)$

$$
\widetilde{v}(\infty)=\frac{2}{\sigma^{2}} \int_{c}^{\infty} y^{-1} e^{\gamma \sqrt{y}}\left(\int_{y}^{\infty} z^{-1} e^{-\gamma \sqrt{z}} d z\right) d y
$$

Since $\gamma>0$, then $\lim _{y \rightarrow \infty} y^{-\frac{1}{2}} e^{-\gamma \sqrt{y}}=0$, and from L'Hôpital's rule

$$
\lim _{y \rightarrow \infty} \frac{\int_{y}^{\infty} z^{-1} e^{-\gamma \sqrt{z}} d z}{y^{-\frac{1}{2}} e^{-\gamma \sqrt{y}}}=\lim _{y \rightarrow \infty} \frac{1}{\frac{1}{2} y^{-1 / 2}+\frac{\gamma}{2}}=\frac{2}{\gamma}>0 .
$$

As $y \rightarrow \infty, \int_{y}^{\infty} z^{-1} e^{-\gamma \sqrt{z}} d z \sim \frac{2}{\gamma} y^{-\frac{1}{2}} e^{-\gamma \sqrt{y}}$, and thus there exists $M<\infty$, such that for $y>M$

$$
\int_{y}^{\infty} z^{-1} e^{-\gamma \sqrt{z}} d z<\frac{4}{\gamma} y^{-\frac{1}{2}} e^{-\gamma \sqrt{y}}
$$

Substitute (64) into (62)

$$
\begin{aligned}
\widetilde{v}(\infty) & =\frac{2}{\sigma^{2}} \int_{c}^{M} y^{-1} e^{\gamma \sqrt{y}}\left(\int_{y}^{\infty} z^{-1} e^{-\gamma \sqrt{z}} d z\right) d y+\frac{2}{\sigma^{2}} \int_{M}^{\infty} y^{-1} e^{\gamma \sqrt{y}}\left(\int_{y}^{\infty} z^{-1} e^{-\gamma \sqrt{z}} d z\right) d y \\
& <\frac{2}{\sigma^{2}} \int_{c}^{M} y^{-1} e^{\gamma \sqrt{y}}\left(\int_{y}^{\infty} z^{-1} e^{-\gamma \sqrt{z}} d z\right) d y+\frac{2}{\sigma^{2}} \int_{M}^{\infty} y^{-1} e^{\gamma \sqrt{y}}\left(\frac{4}{\gamma^{-\frac{1}{2}}} e^{-\gamma \sqrt{y}}\right) d y \\
& =\frac{2}{\sigma^{2}} \int_{c}^{M} y^{-1} e^{\gamma \sqrt{y}}\left(\int_{y}^{\infty} z^{-1} e^{-\gamma \sqrt{z}} d z\right) d y+\frac{8}{\gamma \sigma^{2}} \int_{M}^{\infty} y^{-\frac{3}{2}} d y \\
& <\infty
\end{aligned}
$$

From the definition in (34)

$$
\widetilde{v}_{b}(\infty)=\frac{2}{\sigma^{2}} \int_{c}^{\infty} e^{\gamma \sqrt{y}}\left(\int_{y}^{\infty} z^{-1} e^{-\gamma \sqrt{z}} d z\right) d y,
$$

From (63), there exits $M^{\prime}>c>0$, such that for $y>M^{\prime}$

$$
\int_{y}^{\infty} z^{-1} e^{-\gamma \sqrt{z}} d z>\frac{1}{\gamma} y^{-\frac{1}{2}} e^{-\gamma \sqrt{y}}
$$

Substitute (66) into (65)

$$
\begin{aligned}
\tilde{v}_{b}(\infty) & \geqslant \frac{2}{\sigma^{2}} \int_{M^{\prime}}^{\infty} e^{\gamma \sqrt{y}}\left(\int_{y}^{\infty} z^{-1} e^{-\gamma \sqrt{z}} d z\right) d y \\
& >\frac{2}{\sigma^{2}} \int_{M^{\prime}}^{\infty} e^{\gamma \sqrt{y}}\left(\frac{1}{\gamma} y^{-\frac{1}{2}} e^{-\gamma \sqrt{y}}\right) d y=\frac{2}{\sigma^{2}} \int_{M^{\prime}}^{\infty} y^{-\frac{1}{2}} d y=\infty .
\end{aligned}
$$

(III) $\mu<\frac{1}{2} \sigma^{2}$. We consider the case when $\alpha<1$. Since $-\frac{\alpha+1}{2}>-1$, then from the property of the gamma function

$$
\widetilde{s}(0)=-C_{1} \int_{0}^{c} y^{-\frac{\alpha+1}{2}} e^{-\gamma \sqrt{y}} d y>-\infty
$$


From (56), we have $\widetilde{s}(\infty)=2 C_{1} \int_{\sqrt{c}}^{\infty} z^{-\alpha} e^{-\gamma z} d z$, and divide into three cases. If $\gamma>0$, then from the property of gamma function, $\widetilde{s}(\infty)<\infty$. If $\gamma \leqslant 0$, then $e^{-\gamma z} \geqslant 1$, and $\widetilde{s}(\infty) \geqslant$ $2 C_{1} \int_{\sqrt{c}}^{\infty} z^{-\alpha} d z=\infty$. To summarize, when $\alpha<1$

$$
\widetilde{s}(\infty) \begin{cases}=\infty, & \text { if } \gamma \leqslant 0 \\ <\infty, & \text { if } \gamma>0\end{cases}
$$

We first look at $\widetilde{v}(0)$ and $\widetilde{v}_{b}(0)$. From the definition in (34)

$$
\widetilde{v}(0)=\frac{2}{\sigma^{2}} \int_{0}^{c} y^{\frac{\alpha-3}{2}} e^{\gamma \sqrt{y}}\left(\int_{0}^{y} z^{-\frac{\alpha+1}{2}} e^{-\gamma \sqrt{z}} d z\right) d y
$$

and

$$
\widetilde{v}_{b}(0)=\frac{2}{\sigma^{2}} \int_{0}^{c} y^{\frac{\alpha-1}{2}} e^{\gamma \sqrt{y}}\left(\int_{0}^{y} z^{-\frac{\alpha+1}{2}} e^{-\gamma \sqrt{z}} d z\right) d y
$$

Divide into two cases based on $\gamma$. When $\gamma \leqslant 0, e^{-\gamma \sqrt{z}} \geqslant 1$, then

$$
\begin{aligned}
\widetilde{v}(0) & \geqslant \frac{2}{\sigma^{2}} \int_{0}^{c} y^{\frac{\alpha-3}{2}} e^{\gamma \sqrt{y}}\left(\int_{0}^{y} z^{-\frac{\alpha+1}{2}} d z\right) d y \\
& =\frac{2}{\sigma^{2}} \int_{0}^{c} y^{\frac{\alpha-3}{2}} e^{\gamma \sqrt{y}}\left(\frac{2}{1-\alpha} y^{\frac{1-\alpha}{2}}\right) d y \\
& =\frac{4}{\sigma^{2}(1-\alpha)} \int_{0}^{c} y^{-1} e^{\gamma \sqrt{y}} d y .
\end{aligned}
$$

Apply a change of variable $z=\sqrt{y}$, then

$$
\widetilde{v}(0) \geqslant \frac{4}{\sigma^{2}(1-\alpha)} \int_{0}^{c} y^{-1} e^{\gamma \sqrt{y}} d y=\frac{8}{\sigma^{2}(1-\alpha)} \int_{0}^{\sqrt{c}} z^{-1} e^{\gamma z} d z=\infty .
$$

The last equality is from the property of the gamma function. Then $\widetilde{v}(0)=\infty$ holds when $\gamma \leqslant 0$.

Since $\gamma \leqslant 0$ is assumed, then $e^{-\gamma \sqrt{z}} \leqslant e^{-\gamma \sqrt{y}}$ for $0 \leqslant z \leqslant y$, and

$$
\widetilde{v}_{b}(0) \leqslant \frac{2}{\sigma^{2}} \int_{0}^{c} y^{\frac{\alpha-1}{2}} e^{\gamma \sqrt{y}}\left(\int_{0}^{y} z^{-\frac{\alpha+1}{2}} e^{-\gamma \sqrt{y}} d z\right) d y=\frac{4 c}{\sigma^{2}(1-\alpha)}<\infty .
$$

Then $\widetilde{v}_{b}(0)<\infty$ holds when $\gamma \leqslant 0$.

When $\gamma>0, e^{-\gamma \sqrt{z}}>e^{-\gamma \sqrt{y}}$ for $0 \leqslant z \leqslant y$, then

$$
\widetilde{v}(0)>\frac{2}{\sigma^{2}} \int_{0}^{c} y^{\frac{\alpha-3}{2}} e^{\gamma \sqrt{y}}\left(\int_{0}^{y} z^{-\frac{\alpha+1}{2}} e^{-\gamma \sqrt{y}} d z\right) d y=\frac{4}{\sigma^{2}(1-\alpha)} \int_{0}^{c} y^{-1} d y=\infty .
$$

Then $\widetilde{v}(0)=\infty$ holds when $\gamma>0$. 
When $\gamma>0, e^{-\gamma \sqrt{z}}<1$ for $0 \leqslant z \leqslant y$, then

$$
\begin{aligned}
\widetilde{v}_{b}(0) & =\frac{2}{\sigma^{2}} \int_{0}^{c} y^{\frac{\alpha-1}{2}} e^{\gamma \sqrt{y}}\left(\int_{0}^{y} z^{-\frac{\alpha+1}{2}} e^{-\gamma \sqrt{z}} d z\right) d y \\
& <\frac{2}{\sigma^{2}} \int_{0}^{c} y^{\frac{\alpha-1}{2}} e^{\gamma \sqrt{y}}\left(\int_{0}^{y} z^{-\frac{\alpha+1}{2}} d z\right) d y \\
& =\frac{2}{\sigma^{2}} \int_{0}^{c} y^{\frac{\alpha-1}{2}} e^{\gamma \sqrt{y}}\left(\frac{2}{1-\alpha} y^{\frac{1-\alpha}{2}}\right) d y \\
& =\frac{4}{\sigma^{2}(1-\alpha)} \int_{0}^{c} e^{\gamma \sqrt{y}} d y \\
& <\infty .
\end{aligned}
$$

Then $\widetilde{v}_{b}(0)<\infty$ holds when $\gamma>0$.

To summarize, we have that $\widetilde{v}(0)=\infty$ and $\widetilde{v}_{b}(0)<\infty$ hold when $\alpha<1$.

Consider the case when $\alpha<1$ and $\gamma>0$. From the definition in (34)

$$
\widetilde{v}(\infty)=\frac{2}{\sigma^{2}} \int_{c}^{\infty} y^{\frac{\alpha-3}{2}} e^{\gamma \sqrt{y}}\left(\int_{y}^{\infty} z^{-\frac{\alpha+1}{2}} e^{-\gamma \sqrt{z}} d z\right) d y
$$

and

$$
\widetilde{v}_{b}(\infty)=\frac{2}{\sigma^{2}} \int_{c}^{\infty} y^{\frac{\alpha-1}{2}} e^{\gamma \sqrt{y}}\left(\int_{y}^{\infty} z^{-\frac{\alpha+1}{2}} e^{-\gamma \sqrt{z}} d z\right) d y
$$

Since $\gamma>0$ is assumed, then $\lim _{y \rightarrow \infty} y^{-\frac{\alpha}{2}} e^{-\gamma \sqrt{y}}=0$, and from L'Hôpital's rule

$$
\lim _{y \rightarrow \infty} \frac{\int_{y}^{\infty} z^{-\frac{\alpha+1}{2}} e^{-\gamma \sqrt{z}} d z}{y^{-\frac{\alpha}{2}} e^{-\gamma \sqrt{y}}}=\lim _{y \rightarrow \infty} \frac{1}{\frac{\alpha}{2} y^{-\frac{1}{2}}+\frac{\gamma}{2}}=\frac{2}{\gamma}>0 .
$$

As $y \rightarrow \infty, \int_{y}^{\infty} z^{-\frac{\alpha+1}{2}} e^{-\gamma \sqrt{z}} d z \sim \frac{2}{\gamma} y^{-\frac{\alpha}{2}} e^{-\gamma \sqrt{y}}$, and there exists $M>0$, such that for $y>M$

$$
\int_{y}^{\infty} z^{-\frac{\alpha+1}{2}} e^{-\gamma \sqrt{z}} d z<\frac{4}{\gamma} y^{-\frac{\alpha}{2}} e^{-\gamma \sqrt{y}}
$$

Substitute (72) into (69)

$$
\begin{aligned}
\widetilde{v}(\infty) & =\frac{2}{\sigma^{2}} \int_{c}^{M} y^{\frac{\alpha-3}{2}} e^{\gamma \sqrt{y}}\left(\int_{y}^{\infty} z^{-\frac{\alpha+1}{2}} e^{-\gamma \sqrt{z}} d z\right) d y+\frac{2}{\sigma^{2}} \int_{M}^{\infty} y^{\frac{\alpha-3}{2}} e^{\gamma \sqrt{y}}\left(\int_{y}^{\infty} z^{-\frac{\alpha+1}{2}} e^{-\gamma \sqrt{z}} d z\right) d y \\
& <\frac{2}{\sigma^{2}} \int_{c}^{M} y^{\frac{\alpha-3}{2}} e^{\gamma \sqrt{y}}\left(\int_{y}^{\infty} z^{-\frac{\alpha+1}{2}} e^{-\gamma \sqrt{z}} d z\right) d y+\frac{2}{\sigma^{2}} \int_{M}^{\infty} y^{\frac{\alpha-3}{2}} e^{\gamma \sqrt{y}}\left(\frac{4}{\gamma} y^{-\frac{\alpha}{2}} e^{-\gamma \sqrt{y}}\right) d y \\
& =\frac{2}{\sigma^{2}} \int_{c}^{M} y^{\frac{\alpha-3}{2}} e^{\gamma \sqrt{y}}\left(\int_{y}^{\infty} z^{-\frac{\alpha+1}{2}} e^{-\gamma \sqrt{z}} d z\right) d y+\frac{8}{\gamma \sigma^{2}} \int_{M}^{\infty} y^{-\frac{3}{2}} d y \\
& =\frac{2}{\sigma^{2}} \int_{c}^{M} y^{\frac{\alpha-3}{2}} e^{\gamma \sqrt{y}}\left(\int_{y}^{\infty} z^{-\frac{\alpha+1}{2}} e^{-\gamma \sqrt{z}} d z\right) d y+\frac{16}{\sqrt{M} \gamma \sigma^{2}} \\
& <\infty
\end{aligned}
$$


Then $\widetilde{v}(\infty)<\infty$, for $\alpha<1$ and $\gamma>0$.

From (71), there exists $M^{\prime}>c>0$, such that for $y>M^{\prime}$

$$
\int_{y}^{\infty} z^{-\frac{\alpha+1}{2}} e^{-\gamma \sqrt{z}} d z>\frac{1}{\gamma} y^{-\frac{\alpha}{2}} e^{-\gamma \sqrt{y}}
$$

Substitute (73) into (70) to obtain

$$
\begin{aligned}
\widetilde{v}_{b}(\infty) & \geqslant \frac{2}{\sigma^{2}} \int_{M^{\prime}}^{\infty} y^{\frac{\alpha-1}{2}} e^{\gamma \sqrt{y}}\left(\int_{y}^{\infty} z^{-\frac{\alpha+1}{2}} e^{-\gamma \sqrt{z}} d z\right) d y \\
& >\frac{2}{\sigma^{2}} \int_{M^{\prime}}^{\infty} y^{\frac{\alpha-1}{2}} e^{\gamma \sqrt{y}}\left(\frac{1}{\gamma} y^{-\frac{\alpha}{2}} e^{-\gamma \sqrt{y}}\right) d y=\frac{2}{\gamma \sigma^{2}} \int_{M^{\prime}}^{\infty} y^{-\frac{1}{2}} d y=\infty .
\end{aligned}
$$

Then $\widetilde{v}_{b}(\infty)=\infty$, for $\alpha<1$ and $\gamma>0$.

\section{B.6 Proof of Proposition 5.11}

Proof. From the proof in Proposition 5.10, we study separately the following three cases (I), (II) and (III).

(I) $\mu>\frac{1}{2} \sigma^{2}$. Then we have the following classification:

$$
\widetilde{s}(\infty) \begin{cases}<\infty, & \text { if } \gamma \geqslant 0, \\ =\infty, & \text { if } \gamma<0,\end{cases}
$$

and $\widetilde{s}(0)=-\infty$ for $\gamma \in \mathbb{R}$. This result, combined with the classification in Table 11, gives us the first three rows of Table 12. From Table 12 and Proposition 4.2, we have that when $\mu>\frac{1}{2} \sigma^{2}$, $\left(S_{t}\right)_{0 \leqslant t \leqslant \infty}$ is not a uniformly integrable $P$-martingale.

(II) $\mu=\frac{1}{2} \sigma^{2}$. Then we have the following classification:

$$
\widetilde{s}(\infty) \begin{cases}<\infty, & \text { if } \gamma>0, \\ =\infty, & \text { if } \gamma \leqslant 0\end{cases}
$$

and $\widetilde{s}(0)=-\infty$ for $\gamma \in \mathbb{R}$.

This result, combined with the classification in Table 11, gives us the three middle rows of the classification in Table 12. From Table 12 and Proposition 4.2, we have that when $\mu=\frac{1}{2} \sigma^{2}$, $\left(S_{t}\right)_{0 \leqslant t \leqslant \infty}$ is not a uniformly integrable $P$-martingale.

(III) $\mu<\frac{1}{2} \sigma^{2}$. Then we have the following classification:

$$
\widetilde{s}(\infty) \begin{cases}<\infty, & \text { if } \gamma>0, \\ =\infty, & \text { if } \gamma \leqslant 0\end{cases}
$$

and $\widetilde{s}(0)>-\infty$ for $\gamma \in \mathbb{R}$.

This result, combined with the classification in Table 11, gives us the last three rows of the classification in Table 12. From Table 12 and Proposition 4.2, we have that when $\mu<\frac{1}{2} \sigma^{2}$, $\left(S_{t}\right)_{0 \leqslant t \leqslant \infty}$ is a uniformly integrable $P$-martingale if and only if $\gamma \leqslant 0$, or equivalently $\rho \leqslant 0$. 